\title{
Homocysteine thiolactone and $N$-homocysteinylated protein induce pro-atherogenic changes in gene expression in human vascular endothelial cells
}

\author{
Dorota Gurda $^{1}$ - Luiza Handschuh ${ }^{1} \cdot$ Weronika Kotkowiak $^{1}$. \\ Hieronim Jakubowski ${ }^{1,2,3}$
}

Received: 28 January 2015 / Accepted: 4 March 2015 / Published online: 24 March 2015

(C) The Author(s) 2015. This article is published with open access at Springerlink.com

\begin{abstract}
Genetic or nutritional deficiencies in homocysteine (Hcy) metabolism lead to hyperhomocysteinemia (HHcy) and cause endothelial dysfunction, a hallmark of atherosclerosis. In addition to Hcy, related metabolites accumulate in HHcy but their role in endothelial dysfunction is unknown. Here, we examine how Hcy-thiolactone, $\mathrm{N}$-Hcyprotein, and Hcy affect gene expression and molecular pathways in human umbilical vein endothelial cells. We used microarray technology, real-time quantitative polymerase chain reaction, and bioinformatic analysis with PANTHER, DAVID, and Ingenuity Pathway Analysis (IPA) resources. We identified 47,113 , and 30 mRNAs regulated by $\mathrm{N}$-Hcyprotein, Hcy-thiolactone, and Hcy, respectively, and found that each metabolite induced a unique pattern of gene expression. Top molecular pathways affected by Hcy-thiolactone were chromatin organization, one-carbon metabolism, and lipid-related processes $[-\log (P$ value $)=20-31]$. Top pathways affected by $\mathrm{N}$-Hcy-protein and Hcy were blood coagulation, sulfur amino acid metabolism, and lipid metabolism $[-\log (P$ value $)]=4-11$; also affected by Hcythiolactone, $[-\log (P$ value $)=8-14]$. Top disease related to Hcy-thiolactone, $N$-Hcy-protein, and Hcy was 'atherosclerosis, coronary heart disease' $[-\log (P$ value $)=9-16]$.
\end{abstract}

Handling Editor: G. Lubec.

Hieronim Jakubowski

jakubows@njms.rutgers.edu

Institute of Bioorganic Chemistry, Poznań, Poland

2 Department of Biochemistry and Biotechnology, University of Life Sciences, Poznań, Poland

3 Department of Microbiology, Biochemistry and Molecular Genetics, Rutgers-New Jersey Medical School, International Center for Public Health, 225 Warren Street, Newark, NJ 07101-1709, USA
Top-scored biological networks affected by Hcy-thiolactone (score $=34-40$ ) were cardiovascular disease and function; those affected by $N$-Hcy-protein (score $=24-35$ ) were 'small molecule biochemistry, neurological disease,' and 'cardiovascular system development and function'; and those affected by Hcy (score = 25-37) were 'amino acid metabolism, lipid metabolism,' 'cellular movement, and cardiovascular and nervous system development and function.' These results indicate that each Hcy metabolite uniquely modulates gene expression in pathways important for vascular homeostasis and identify new genes and pathways that are linked to HHcy-induced endothelial dysfunction and vascular disease.

Keywords Endothelial cells · Homocysteine metabolites . Gene expression $\cdot$ Microarrays $\cdot$ Bioinformatic analysis

$\begin{array}{ll}\text { Abbreviations } \\ \text { CBS } & \text { Cystathionine } \beta \text {-synthase } \\ \text { CVD } & \text { Cardiovascular disease } \\ \text { Fbg } & \text { Fibrinogen } \\ \text { Hcy } & \text { Homocysteine } \\ \text { HHcy } & \text { Hyperhomocysteinemia } \\ \text { HUVECs } & \text { Human umbilical vein endothelial cells } \\ \text { IPA } & \text { Ingenuity pathway analysis } \\ \text { RT-qPCR } & \begin{array}{l}\text { Real-time quantitative polymerase chain } \\ \text { reaction }\end{array}\end{array}$

\section{Introduction}

Homocysteine (Hcy) is an important intermediate in onecarbon metabolism that involves the conversion of methionine to cysteine (Mudd et al. 2001) and folate interconversions (Strauss et al. 2007). Deficiencies in these pathways 
lead to the accumulation of Hcy and its metabolites in the blood-hyperhomocysteinemia (HHcy) — which is an independent risk factor for cardiovascular disease (CVD) and causes endothelial dysfunction, a hallmark of atherosclerosis (Dayal and Lentz 2008). However, molecular mechanisms underlying the pathophysiology of HHcy are not fully understood (Jakubowski 2011, 2013; Perla-Kajan et al. 2007). One hypothesis states that metabolic conversion of Hcy to Hcythiolactone initiates a pathway that leads to pathologies associated with HHcy (Jakubowski 1997a, 1999, 2007). Hcy-thiolactone is chemically reactive and modifies $\varepsilon$-amino groups of protein lysine residues, which generates immunogenic and toxic $N$-homocysteinylated protein ( $N$-Hcy-protein) (Jakubowski 2008, 2013; Jakubowski et al. 2000).

In humans and mice, HHcy leads to the accumulation of Hcy-thiolactone and $N$-Hcy-protein, in addition to Hcy (Chwatko et al. 2007; Jakubowski et al. 2008, 2009). We and other investigators have shown that HHcy induces changes in gene expression in mouse models that are associated with atherothrombotic disease (Devlin et al. 2005; DiBello et al. 2010; Ingrosso et al. 2003; Kim et al. 2011; Pogribny et al. 2008; Sharma et al. 2006; Suszynska-Zajczyk et al. 2014a, b, c, d). However, it is not known what mechanism(s) are involved and which metabolite-Hcy itself, Hcy-thiolactone, or $\mathrm{N}$-Hcy-protein-is responsible for changes in gene expression.

The key to understanding mechanisms by which HHcy disrupts normal cellular function and ultimately causes disease is to identify genes whose expression is affected by individual Hcy metabolites. It is likely that each metabolite causes specific alterations in gene expression that individually contribute to endothelial dysfunction induced by HHcy. Defining metabolite-specific alterations in gene expression in endothelial cells will help to identify molecular pathways involved in the pathology of HHcy and to uncover novel pharmacologic targets for preventing or treating cardiovascular and neurological disorders of HHcy.

An analysis of genes and biological pathways responding to specific Hcy metabolites has not been previously reported. To determine whether changes in gene expression are metabolite-specific and to identify biological pathways altered by HHcy, we studied how treatments with Hcy-thiolactone, $\mathrm{N}$-Hcy-protein, and Hcy affect gene expression in human umbilical vein endothelial cells (HUVECs), a frequently used model of vascular cells.

\section{Materials and methods}

\section{Cell culture and treatments}

HUVECs, purchased from Lonza, were seeded into $75-\mathrm{cm}^{2}$ flasks coated with $1 \%$ gelatin and cultured in EGM2 medium (Lonza) at $37^{\circ} \mathrm{C} / 5 \% \mathrm{CO}_{2}$, supplemented with $2 \%$ fetal bovine serum (FBS; Sigma-Aldrich), bovine endothelial cell growth factor, and antibiotics: $50 \mu \mathrm{g} / \mathrm{mL}$ penicillin and $100 \mu \mathrm{g} / \mathrm{mL}$ streptomycin (Sigma-Aldrich). Cells between 4 and 10 passage were used in the experiments. After reaching 80-90\% confluence, the cells were treated for $24 \mathrm{~h}$ with 0,10 , or $1000 \mu \mathrm{M} \mathrm{D}$, L-Hcy, or L-Hcy-thiolactone $\mathrm{HCl}$ (Sigma-Aldrich), 10 or $40 \mu \mathrm{M} \mathrm{N}$-Hcy-protein in the EGM2/FBS medium without antibiotics.

\section{$N$-Hcy-protein preparation}

$N$-Hcy-protein was prepared by the modification of FBS with Hcy-thiolactone (Jakubowski 1999). Such preparation mimics $N$-Hcy-protein that was present in the plasma of cystathionine $\beta$-synthase (CBS) and methylenetetrahydrofolate reductase (MTHFR)-deficient patients (Jakubowski et al. 2008). FBS ( $0.2 \mathrm{~mL}, 50 \mathrm{mg}$ protein $/ \mathrm{ml})$ was incubated with 5 or $25 \mathrm{mM} \mathrm{L-Hcy-thiolactone \cdot HCl} \mathrm{(Sigma-Aldrich)}$ in $0.1 \mathrm{M}$ phosphate buffer pH 7.4, $1 \mathrm{mM}$ EDTA (buffer A) at $37^{\circ} \mathrm{C}$ for $16 \mathrm{~h}$. Hcy and unreacted Hcy-thiolactone were removed by four cycles of dilution with buffer A containing $2 \mathrm{mM}$ dithiothreitol (DTT) and ultrafiltration using a 10-kDa cutoff Millipore device (10,000xg, $\left.4{ }^{\circ} \mathrm{C}, 30 \mathrm{~min}\right)$. DTT was then removed by three cycles of dilution with $0.2 \mathrm{~mL}$ buffer A and ultrafiltration, and the $N$-Hcy-protein preparation was diluted to $50 \mathrm{mg}$ protein $/ \mathrm{mL}$ with buffer A. The thiol content in $\mathrm{N}$-Hcy-protein was quantified according to Ellman (1959). These $\mathrm{N}$-Hcy-protein preparations were added to cell culture media to constitute $2 \%$ of the medium volume, resulting in $\mathrm{N}$-Hcy-protein concentrations of 10.1 and $41.1 \mu \mathrm{M}$, respectively, similar to the range of $\mathrm{N}$-Hcy-protein concentrations observed in disorders of Hcy/folate metabolism in humans (Jakubowski et al. 2008) and mice (Jakubowski et al. 2009).

\section{Protein thiols quantification}

The thiol content in $\mathrm{N}$-Hcy-protein was quantified colorimetrically at $412 \mathrm{~nm}$ by using 5,5'-dithiobis-2-nitrobenzoic acid (Sigma-Aldrich) and a molar extinction coefficient 13,600 $\mathrm{M}^{-1} \mathrm{~cm}^{-1}$ for 5-nitrothiobenzoate according to Ellman (1959). After modification of serum protein with $0.2 \mathrm{mM}$ Hcy-thiolactone, the protein thiol content increased to 1.18 from a reference value of 1.0 in untreated serum protein. Increasing Hcy-thiolactone concentration to 1,5 , and 25 increased the thiol content in $N$-Hcy-protein to $1.72,2.34$, and 3.74 thiol groups, respectively. Since albumin with its single Cys34 free thiol group is the major contributor to serum protein thiol content (Glowacki and Jakubowski 2004), these values indicate that the modification with Hcy-thiolactone afforded $0.18,0.72,1.34$, and 2.74 mol $N$-linked Hcy per mol serum protein (albumin). 


\section{RNA extraction}

Total RNA enriched in small RNA ( $>200$ bp) was isolated from HUVECs treated with Hcy-thiolactone, $N$-Hcy-protein, Hcy, or untreated control cells using miRvana miRNA Isolation Kit (Life Technologies), according to the supplier's protocol. Total RNA concentration was measured by using Nano Drop UV/Vis spectrophotometer (2000c, Thermo Scientific). Total RNA quality was assessed by capillary electrophoresis with 2100 Agilent Bioanalyzer (Agilent Technologies). Only high-quality total RNA (RIN > 8.0) was used in the experiments.

\section{cDNA microarray procedures}

Total RNA (20 $\mu \mathrm{g})$ was reverse-transcribed to cDNA using aminoallyl-modified nucleotides. In the next step, cDNA was fluorescently labeled (Superscript Indirect cDNA Labeling System, Invitrogen) according to the manufacturer's protocol. Control cDNA was labeled with Alexa 555, while cDNA prepared from RNA isolated from Hcy-, HTL-, or $N$-Hcy-protein-treated HUVECs was labeled with Alexa 647. The amount of linked fluorescent dye was measured by Nano Drop UV/Vis spectrophotometer (2000c, Thermo Scientific). Only samples with at least $100 \mathrm{pmol}$ of the dye linked were used in the assay. Each pair of cDNA samples (from control and treated cells) was mixed 1:1 with hybridization buffer (500 mM NaH2PO4, $2 \%$ SDS, $2 \mathrm{mM}$ EDTA, $2 \times \mathrm{SSC})$, denatured $\left(95{ }^{\circ} \mathrm{C}, 3 \mathrm{~min}\right)$, kept at $42{ }^{\circ} \mathrm{C}$, and transferred onto OneArray Human Microarrays V4 (Phalanx Biotech Group). Directly before use, microarrays were cross-linked using DNA Crosslinker CL E508 $\mathrm{G}$ (Uvitec, $0,18 \mathrm{~J}$ ), baked at $60{ }^{\circ} \mathrm{C}$ for $10 \mathrm{~min}$ in SciGene hybridization oven (Oxford Gene Technology), washed with $100 \%$ ethanol (15-s hold, 20-s shake), and deionized with water. Pre-hybridization was carried out in a prewarmed $\left(50{ }^{\circ} \mathrm{C}\right)$ buffer containing $250 \mathrm{mM}$ ethanolamine, $0.1 \%$ SDS, and $200 \mathrm{mM} \mathrm{Na}_{2} \mathrm{CO}_{3}(1 \mathrm{~h}, \mathrm{RT})$. The slides were then washed with $80 \%$ ethanol/10 $\mathrm{mM} \mathrm{Na}_{2} \mathrm{CO}_{3}$ and water, centrifuged, and incubated with the labelled cDNA introduced under the coverslips $(24 \times 60 \mathrm{~mm}$, Menzel Glaser) in a chambered box in the hybridization oven $\left(40{ }^{\circ} \mathrm{C}\right.$, 16-18 h). Following hybridization, the slides were washed three times using a microarray ArrayIt wash station [(1) $1 \times \mathrm{SSPE} / 0.03 \% \mathrm{SDS}, 2 \mathrm{~min}, 42{ }^{\circ} \mathrm{C}$; (2) $1 \times \mathrm{SSPE}, 2 \mathrm{~min}$, RT; (3) $0.1 \times$ SSPE, RT], dried by centrifugation, and scanned with Axon 4200AL microarray scanner (Molecular Devices). Hybridizations were carried out in duplicate.

\section{Microarray data analysis}

Microarray data analysis was carried out as previously described (Schmidt et al. 2011). Microarray images were processed using GenePix Pro version 6.1 software (molecular devices). Raw data (gpr files) were submitted to a normalization procedure using R Bioconductor software version 2.13.1 (Smyth and Speed 2003) and limma package (Smyth and Speed 2003). Within-array normalization was carried out using Loess method, while between-array normalization was carried using Aquantile method. To quantify changes in gene expression, the normalized microarray data were fitted to linear models (Smyth 2004). The final $P$ values were adjusted using the FDR correction (Benjamini and Hochberg 1995). Adjusted $P$ value $<0.05$ was considered significant.

\section{Real-time qPCR}

cDNA synthesis was carried out using $1 \mu \mathrm{g}$ of total RNA and Riveraid H Minus First Strand Synthesis cDNA Kit (Thermo Scientific). Quantitative real-time PCR analysis was carried out in Mx 3005P qPCR System (Agilent Technologies) using DyNAmo HS SYBR Green qPCR Kit (Finnzymes) according to the manufacturer's protocol. Primers were purchased from GenoMed S.A. (Warsaw, Poland); the sequences are shown in Table 1. Relative expression was calculated using the $-\Delta \Delta \mathrm{C}_{t}$ method using the SYBR Green fluorescence. A reference gene was HPRT. Each reaction was carried out in duplicate (technical replication) for three separate HUVEC cultures (biological replications).

\section{Pathway and network analysis}

Bioinformatic analysis to discover biological pathways and human diseases linked to genes responding to Hcythiolactone, $\mathrm{N}$-Hcy-protein, and Hcy was carried out using the PANTHER classification system (http://www.pantherdb.org/) and DAVID Knowledgebase and functional annotation tools (http://david.niaid.nih.gov). The networks containing those genes were identified using the Ingenuity Pathway Analysis software (IPA, Ingenuity Systems, Mountain View, CA). The data sets containing differentially expressed mRNAs identified in microarray and RTqPCR experiments were uploaded into the IPA Knowledge database, which mapped gene/gene products to global molecular networks to identify genes that are known to interact with other gene products in the database.

\section{Statistical analysis}

RT-qPCR results are expressed as a mean \pm SD. One-way analysis of variance was used to determine the significance of RT-qPCR experiments (GraphPad Prism Software). Fisher's exact test was used to determine $P$ values in the pathway and network analyses. In all cases, $P$ values $<0.05$ were considered significant. 
Table 1 List of primers used for RT-qPCR

\begin{tabular}{|c|c|c|c|}
\hline Gene name & Accession number & Forward primer & Reverse primer \\
\hline$C B S$ & 875 & GCCAGGTTCGACTCCCCGGA & GCTGGCGTTGCGGTACTGGT \\
\hline$C D 9$ & 928 & CTTCTGGCTTGCCGGGATTGCT & GCGCCGGCTCCGATCAGAAT \\
\hline ESM1 & 11,082 & GCGGTGGACTGCCCTCAACA & CCAGATGCCATGTCATGCTCTTTGC \\
\hline OS9 & 10,956 & GCTGGCATGGAGCGGGAACT & TCTGTGCTCAGGATCCTCCTCTGT \\
\hline MTR & 4548 & CTGCCAATGCCAAGGCAGCCT & GTCCGGAAAGAGTCCGCCCAC \\
\hline MTRR & 4552 & CTGGCGCAAGGTTGGTGGAAGT & TAACAGTGAAACCACGCCGCGC \\
\hline ANXA8 & 653,145 & TGGGAAAGGTGCCCCCGAGG & GCCTGCTCGTTGGTCCCGAT \\
\hline APOL1 & 8542 & GGCTGTGCTGTGTCCCTAATGGG & CACAGGCACCATTCTGCAACGC \\
\hline APOA5 & 116,519 & GCCTCCСТCCACCTGTCTTCTCAGA & AGTCCCAGAAGCCTTTCCGTGC \\
\hline$M A R C H 1$ & 55,016 & AGAAGTGTGCATAACCATGAGTGGG & TCTGCCTGTCCCTTGCGTCA \\
\hline$V E G F A$ & 7422 & GGTGCCCGCTGCTGTCTAATG & TCAAGCTGCCTCGCCTTGCAAC \\
\hline MMP19 & 4327 & GCACGGACAGCCTCTGGAGC & ACAGGTAGTCCACGGGCGCC \\
\hline NTN4 & 59,277 & CGGAGGAGGACGCCCAGGAG & GGAGCCCCGGGACCATAGCC \\
\hline DDX28 & 55,794 & GTCCGCAGTCCCGACGAACC & GTCGAACCAGCACCGGCCTC \\
\hline$S C U B E 1$ & 80,274 & CGGGCTTGGTCTGCCTGGTG & CAGGCTGGTACGTGCCCACG \\
\hline ROBLD3 & 28,956 & CGCCTACGACCGGAACGGGA & ACCAAAGCCTGGGCCATGCAG \\
\hline SLC44A3 & 126,969 & GGGCGCCGAGTACCTGGTTTC & CCAGCCACCACCGAGTAGCC \\
\hline ST8SIA2 & 8128 & ATCGTGGGCAACTCGGGGGT & AAGGCCCGCTGGATGACCGA \\
\hline NCEH1 & 57,552 & GAAGAGCCACTGAAACGCAGCG & GACCAGGACGCACTTGCACTTG \\
\hline$H P R T$ & 3251 & CTGAGGATTTGGAAAGGGTG & AATCCAGCAGGTCAGCAAAG \\
\hline
\end{tabular}

\section{Results}

\section{Identification of genes affected by Hcy and its metabolites in human endothelial cells}

The Phalanx Biotech OneArray Human Microarrays version 4, containing 2,918,760 human genome probes and 1088 experimental control probes, was used to interrogate 31,741 human genes in HUVECs treated with low and high concentrations of Hcy-thiolactone, $N$-Hcy-protein, and Hcy. Of these genes, 110 were significantly altered in response to Hcy-thiolactone treatment (Table 2), 30 mRNAs were altered in response to $\mathrm{N}$-Hcy-protein (Table 3), and 14 mRNAs were altered in response to Hcy (Table 4). In general, the magnitude of the expression change increased at high metabolite concentrations. Additionally, 3, 17, and 16 genes whose expression was altered by Hcy-thiolactone, $N$-Hcy-protein, or Hcy, respectively were identified by RTqPCR (Fig. 1).

\section{Limited overlap between groups of genes regulated by $N$-Hcy-protein, Hcy-thiolactone, and Hcy}

Only a minority of Hcy-thiolactone-affected genes was also affected by Hcy ( 28 out of 113 ) or by $N$-Hcy-protein ( 22 out of 113). Likewise, only some of $N$-Hcy-protein-regulated genes were also regulated by Hcy-thiolactone (22 out of 47) or Hcy (22 out of 47) (Fig. 2). However, the majority of genes responding to Hcy also responded to Hcy-thiolactone (28 out of 30) and $\mathrm{N}$-Hcy-protein (22 out of 30). There were genes that responded only to one metabolite: Hcy-thiolactone (87 genes), $\mathrm{N}$-Hcy-protein (25 genes), or Hcy (2 genes). Twentytwo genes responded to all three metabolites (Table 5).

Hcy-thiolactone, $N$-Hcy-protein, and Hcy regulated 22 common genes: seven were down-regulated (DDX28, LRRIQ1, MMP19, NCEH1, PHACTR4, SCUBE1, ST8SIA2) and eight were up-regulated (AHCY, APOL1, MTR, MTRR, ESM1, CBS, CD9, MARCH1). Seven other genes were regulated in the opposite direction by $N$-Hcy-protein, compared to Hcy and Hcy-thiolactone: APOA5, ANXA8, NTN4, OS9, $S L C 44 A 3$, and VEGFA were up-regulated by $N$-Hcy-protein, but down-regulated by Hcy and Hcy-thiolactone, while LAMTOR 2 was down-regulated by $\mathrm{N}$-Hcy-protein and upregulated by Hcy and Hcy-thiolactone. Hcy-thiolactone and Hcy down-regulated four genes (CD209, KRT27, LMX1A, $P C D H B 7)$ and up-regulated two (IFI30, SLC22A9).

\section{Genes affected by Hcy-thiolactone}

Of the 113 genes affected by Hcy-thiolactone treatment, seventy-one were up-regulated and forty-two were downregulated (Table 2). Significant changes in gene expression occurred in endothelial cells treated with $10 \mu \mathrm{M}$ Hcy-thiolactone and were enhanced at $1000 \mu \mathrm{M}$ Hcy-thiolactone. The up-regulation varied from 2.02-fold for the regulator of angiogenesis $E P C l$ gene to 22.06-fold for the 
Table 2 List of Hcy-thiolactone-responsive genes and their expression levels

\begin{tabular}{|c|c|c|c|c|}
\hline \multirow[t]{2}{*}{ Gene name } & \multirow[t]{2}{*}{ Accession number } & \multirow[t]{2}{*}{ Protein name } & \multicolumn{2}{|c|}{ Rel. expression, fold } \\
\hline & & & $\begin{array}{l}10 \mu \mathrm{M} \\
\text { Hcy-thiolactone }\end{array}$ & $\begin{array}{l}1000 \mu \mathrm{M} \\
\text { Hcy-thiolactone }\end{array}$ \\
\hline$A D S S L 1$ & 122,622 & Adenylosuccinate synthase like 1 & 2.08 & 3.13 \\
\hline$A H C Y$ & 191 & Adenosylhomocysteinase & 3.63 & 4.29 \\
\hline$A L D H 1 L 1$ & 10,840 & Aldehyde dehydrogenase 1 family, member L1 & 2.98 & 7.92 \\
\hline AMOTL2 & 51,421 & Angiomotin like 2 & 3.24 & 9.48 \\
\hline$A N G P T 2$ & 285 & Angiopoietin 2 & 2.64 & 2.16 \\
\hline ANGPT4 & 51,378 & Angiopoietin 4 & 2.55 & 3.12 \\
\hline ANGPTL3 & 27,329 & Angiopoietin-like 3 & -2.16 & -2.71 \\
\hline ANTXR2 & 118,429 & Anthrax toxin receptor 2 & 1.59 & 4.8 \\
\hline ANXA8 & 653,145 & Annexin A8 & -2.21 & -4.66 \\
\hline$A P O A 1 B$ & 128,240 & Apolipoprotein A-I binding protein & 3.73 & 2.86 \\
\hline APOA4 & 337 & Apolipoprotein A-IV & 2.68 & 6.23 \\
\hline APOA4 & 107,690 & Apolipoprotein A-IV & 2.68 & 2.68 \\
\hline APOA5 & 116,519 & Apolipoprotein A-V & -2.59 & -7.07 \\
\hline$A P O B$ & 338 & Apolipoprotein B & 2.4 & 5.29 \\
\hline APOL1 & 8542 & Apolipoprotein L1 & 3.79 & 5.86 \\
\hline APOL5 & 80,831 & Apolipoprotein L5 & 2.3 & 4.93 \\
\hline APOL6 & 80,830 & Apolipoprotein L6 & 2.58 & 3.32 \\
\hline$A P O L D 1$ & 81,575 & Apolipoprotein L domain containing 1 & -1.13 & -5.56 \\
\hline$A P O O$ & 79,135 & Apolipoprotein $\mathrm{O}$ & 2.27 & 4.82 \\
\hline$B R D 8$ & 10,902 & Bromodomain containing 8 & -2.79 & -4.78 \\
\hline$B T N 3 A 2$ & 11,118 & Butyrophilin, subfamily 3 , member A2 & 1.61 & 2.9 \\
\hline ClQTNF4 & 114,900 & $\mathrm{C} 1 \mathrm{q}$ and tumor necrosis factor related protein 4 & -3.18 & -3.52 \\
\hline$C B S$ & 875 & Cystathionine $\beta$-synthase & 4.73 & 10.6 \\
\hline$C B X 2$ & 12,416 & Chromobox 2 & -5.97 & -7.06 \\
\hline CCDC129 & 223,075 & Coiled-coil domain containing 129 & -2.51 & -2.14 \\
\hline CD209 & 30,835 & CD209 molecule & -2.1 & -4.28 \\
\hline$C D 9$ & 928 & CD9 molecule & 3.55 & 11.75 \\
\hline $\mathrm{CH} 25 \mathrm{H}$ & 9023 & Cholesterol 25-hydroxylase & 1.32 & 2.53 \\
\hline CRSP7 & 9441 & Mediator complex subunit 26 & 1.68 & 4.4 \\
\hline CYBASC3 & 220,002 & Cytochrome b561 family, member A3 & 2.01 & 3.63 \\
\hline$C Y P 2 S 1$ & 29,785 & Cytochrome P450, family 2 , subfamily S, polypeptide 1 & 2.74 & 6.66 \\
\hline DDIT4L & 115,265 & DNA-damage-inducible transcript 4-like & 1.39 & 3.46 \\
\hline DOTIL & 84,444 & DOT1-like, histone H3 methyltransferase & -2.52 & -2.19 \\
\hline EHMT1 & 79,813 & Euchromatic histone-lysine $N$-methyltransferase 1 & 2.02 & 3.69 \\
\hline EHMT2 & 10,919 & Euchromatic histone-lysine $N$-methyltransferase 2 & 1.69 & 2.46 \\
\hline ELP2 & 55,250 & Elongator acetyltransferase complex subunit 2 & 2.09 & 2.41 \\
\hline EP300 & 2033 & E1A binding protein $\mathrm{p} 300$ & 2.06 & 2.79 \\
\hline EPC1 & 80,314 & Enhancer of polycomb homolog 1 & 1.77 & 2.02 \\
\hline ESM1 & 11,082 & Endothelial cell-specific molecule 1 & 2.25 & 6.59 \\
\hline$E Z H 2$ & 2146 & Enhancer of zeste homolog 2 & 2.31 & 5.36 \\
\hline$F A B P 3$ & 2170 & FABP3 fatty acid binding protein 3 , muscle and heart & 3.73 & 4.97 \\
\hline$F G B$ & 2244 & Fibrinogen beta chain & -2.36 & -2.45 \\
\hline$H 2 A F Y$ & 9555 & H2A histone family, member $\mathrm{Y}$ & 1.71 & 2.46 \\
\hline$H D L B P$ & 3069 & High-density lipoprotein binding protein & -1.29 & -4.57 \\
\hline HERPUD2 & 64,224 & $\begin{array}{l}\text { Homocysteine-responsive endoplasmic reticulum-resident } \\
\text { ubiquitin-like domain member } 2\end{array}$ & 4.61 & 5.79 \\
\hline
\end{tabular}


Table 2 continued

\begin{tabular}{|c|c|c|c|c|}
\hline \multirow[t]{2}{*}{ Gene name } & \multirow[t]{2}{*}{ Accession number } & \multirow[t]{2}{*}{ Protein name } & \multicolumn{2}{|c|}{ Rel. expression, fold } \\
\hline & & & $\begin{array}{l}10 \mu \mathrm{M} \\
\text { Hcy-thiolactone }\end{array}$ & $\begin{array}{l}1000 \mu \mathrm{M} \\
\text { Hcy-thiolactone }\end{array}$ \\
\hline HIST1H1B & 3009 & Histone cluster $1, \mathrm{H} 1 \mathrm{~b}$ & -7.7 & -9.38 \\
\hline HISTIH2AJ & 8330 & Histone cluster $1, \mathrm{H} 2 \mathrm{ak}$ & -2.73 & -4.12 \\
\hline HIST1H2BK & 85,236 & Histone cluster $1, \mathrm{H} 2 \mathrm{bk}$ & 2.26 & 2.46 \\
\hline HPSE2 & 60,495 & Heparanase 2 & 2.73 & 6.64 \\
\hline$I B R D C 2$ & 255,488 & E3 Ubiquitin-protein ligase IBRDC2 & -4.24 & -3.94 \\
\hline ICAM1 & 3383 & Intercellular adhesion molecule 1 & 2.56 & 17.3 \\
\hline IFI30 & 10,437 & Interferon gamma-inducible protein 30 & 1.11 & 3.7 \\
\hline IL2ORA & 53,832 & Interleukin 20 receptor, Ralpha & -2.18 & -2.14 \\
\hline IRF2 & 3660 & Interferon regulatory factor 2 & -2.3 & -3.75 \\
\hline JARID2 & 3720 & jumonji, AT rich interactive domain 2 & 3.57 & 2.7 \\
\hline$J M J D 2 B$ & 23,030 & Lysine (K)-specific demethylase 4B & -1.87 & -2.98 \\
\hline KRT27 & 342,574 & Keratin 27 & -2.55 & -6.45 \\
\hline LAMTOR 2 & 28,956 & Late endosomal/lysosomal adaptor, MAPK and MTOR activator 2 & 2.24 & 3.65 \\
\hline$L M X 1 A$ & 4009 & LIM Homeobox transcription factor 1, alpha & -2.14 & -6.27 \\
\hline$L P L$ & 4023 & Lipoprotein lipase & 1.61 & 4.03 \\
\hline$L R P 8$ & 7804 & $\begin{array}{l}\text { Low-density lipoprotein receptor-related protein } 8 \text {, } \\
\text { apolipoprotein e receptor }\end{array}$ & 3.49 & 5.02 \\
\hline LRRIQ1 & 84,125 & Leucine-rich repeats and IQ motif containing 1 & -5.97 & -8.91 \\
\hline MAT1A & 4143 & Methionine adenosyltransferase I & 4.46 & 22.06 \\
\hline MARCH1 & 55,016 & $\begin{array}{l}\text { Membrane-associated ring finger (C3HC4) 1, E3 ubiquitin-protein } \\
\text { ligase }\end{array}$ & 4.14 & 5.46 \\
\hline$M B$ & 4151 & Myoglobin & 4.22 & 18.66 \\
\hline$M B D 3$ & 53,615 & Methyl-CpG-binding domain protein 3 & 1.54 & 2.39 \\
\hline MMP19 & 4327 & Matrix metallopeptidase 19 & -2.45 & -12.75 \\
\hline$M M R N 1$ & 22,915 & Multimerin 1 & -2.68 & -6.25 \\
\hline MSR1 & 4481 & Macrophage scavenger receptor 1 & 3.43 & 2.15 \\
\hline MTHFD2 & 10,797 & $\begin{array}{l}\text { Methylenetetrahydrofolate dehydrogenase } 2 \text {, } \\
\text { methenyltetrahydrofolate cyclohydrolase }\end{array}$ & 2.2 & 4.58 \\
\hline$M T R$ & 4548 & 5-Methyltetrahydrofolate-homocysteine methyltransferase & 2.84 & 15.42 \\
\hline MTRR & 4552 & 5-Methyltetrahydrofolate-homocysteine methyltransferase reductase & 2.31 & 6.27 \\
\hline NCEHI & 57,552 & Neutral cholesterol ester hydrolase 1 & 2.64 & 9.95 \\
\hline NSD1 & 64,324 & Nuclear receptor binding SET domain protein 1 & 2.52 & 3.16 \\
\hline NT5DC4 & 284,958 & $5^{\prime}$-Nucleotidase domain containing 4 & 1.26 & 3.9 \\
\hline NTN4 & 59,277 & Netrin 4 & -2.87 & -12.24 \\
\hline PAPPA2 & 60,676 & Pappalysin 2 & 1.58 & 2.99 \\
\hline PCDHB7 & 56,129 & Protocadherin beta 7 & -2.33 & -5.52 \\
\hline$P F K$ & $5211 \mathrm{~L}$ & Phosphofructokinase, liver & -2.48 & -2.72 \\
\hline PHACTR & 465,979 & Phosphatase and actin regulator 4 & -2.47 & -8.93 \\
\hline$P I G L$ & 9487 & Phosphatidylinositol glycan Anchor biosynthesis, class L & -2.73 & -2.31 \\
\hline$P L G$ & 5340 & Plasminogen & 2.76 & 6.76 \\
\hline$P P A P 2 B$ & 8613 & Phosphatidic acid phosphatase type $2 \mathrm{~B}$ & 3.73 & 8.05 \\
\hline$P P A P 2 C$ & 607,126 & Phosphatidic acid phosphatase type $2 \mathrm{C}$ & 2.41 & 6.29 \\
\hline PTPN6 & 5777 & Protein tyrosine phosphatase, non-receptor type 6 & -3.13 & -12.12 \\
\hline$P T P R B$ & 5787 & Protein tyrosine phosphatase, receptor type, B & -4.07 & -16.67 \\
\hline$S C A R F 1$ & 8578 & Scavenger receptor class $\mathrm{F}$, member 1 & 1.51 & 3.65 \\
\hline$S C L 44 A 3$ & 126,969 & Solute carrier family 44, member 3 & -1.27 & -5.32 \\
\hline SCUBE1 & 80,274 & Signal peptide, CUB domain, EGF-like 1 & -2.19 & -12.37 \\
\hline
\end{tabular}


Table 2 continued

\begin{tabular}{|c|c|c|c|c|}
\hline \multirow[t]{2}{*}{ Gene name } & \multirow[t]{2}{*}{ Accession number } & \multirow[t]{2}{*}{ Protein name } & \multicolumn{2}{|c|}{ Rel. expression, fold } \\
\hline & & & $\begin{array}{l}10 \mu \mathrm{M} \\
\text { Hcy-thiolactone }\end{array}$ & $\begin{array}{l}1000 \mu \mathrm{M} \\
\text { Hcy-thiolactone }\end{array}$ \\
\hline$\overline{\text { SERPINA9 }}$ & 327,657 & $\begin{array}{l}\text { Serpin peptidase inhibitor, clade A (alpha-1 antiproteinase, } \\
\text { antitrypsin), member } 9\end{array}$ & 3.67 & 12.73 \\
\hline SETD2 & 29,072 & Histone-lysine $N$-methyltransferase SETD2 (EC 2.1.1.43) & 17.93 & 6.08 \\
\hline SETD7 & 80,854 & Lysine methyltransferase 7 & 1.07 & 3.9 \\
\hline SHMT1 & 6470 & Serine hydroxymethyltransferase 1 & -1.13 & -2.43 \\
\hline$S L C 22 A 9$ & 114,571 & Solute carrier family 22 (organic anion transporter), member 9 & 2.45 & 4.42 \\
\hline$S L C 2 A 12$ & 154,091 & Solute carrier family 2 (facilitated glucose transporter), member 12 & 2.19 & 2.45 \\
\hline SMOC2 & 64,094 & SPARC related modular calcium binding 2 & 1.59 & 3.02 \\
\hline SMYD3 & 64,754 & SET and MYND domain containing 3 & -2.75 & -11.25 \\
\hline SUV42OH2 & 84,787 & Suppressor of variegation 4-20 homolog 2 & -1.89 & -3.33 \\
\hline THAP1 & 55,145 & THAP domain containing, apoptosis associated protein 1 & 2.72 & 9.99 \\
\hline TLE2 & 7089 & Transducin-like enhancer of split 2 & 1.07 & 3.17 \\
\hline TNFRSF $12 A$ & 51,330 & Tumor necrosis factor receptor superfamily, member $12 \mathrm{~A}$ & 2.07 & 4.19 \\
\hline TNNI3 & 7137 & Troponin I type 3 (cardiac) & 2.22 & 2.66 \\
\hline TNNT2 & 7139 & Troponin T type 2 (cardiac) & 3.27 & 9.67 \\
\hline$U B E 2 G 1$ & 7326 & Ubiquitin-conjugating enzyme E2G 1 & 1.79 & 2.36 \\
\hline USP11 & 8237 & Ubiquitin-specific peptidase 11 & -4.13 & -6.17 \\
\hline$V A V 3$ & 10,451 & Vav 3 guanine nucleotide exchange factor & 4.58 & 13.84 \\
\hline VCAM1 & 7412 & Vascular cell adhesion molecule 1 & 2.17 & 4.31 \\
\hline$V E G F A$ & 7422 & Vascular endothelial growth factor A & -4.37 & -7.37 \\
\hline$V W F$ & 7450 & Von Willebrandt factor & 3.67 & 6.44 \\
\hline ZNF135 & $1,357,694$ & Zinc finger protein 135 & -1.21 & -3.39 \\
\hline
\end{tabular}

$S$-adenosylmethionine synthase MATIA gene. The downregulation was from -2.14-fold for the interleukin-20 receptor subunit alpha IL20RA gene to -16.67 -fold for the protein tyrosine phosphatase beta $P T P R B$ gene.

Genes up-regulated by Hcy-thiolactone include those involved in chromatin modification/assembly, histone methylation/transcription (H2AFY, HIST1H2BK, CRSP7, SETD7, EHMT1, EHMT2, EPC1, NSD1, SETD2, EZH2, EP300, JARID2, ELP2, MBD3), folate and one-carbon metabolism (MAT1A, AHCY, MTR, MTRR, CBS, ALDH1L1, MTHFD2), lipid transport (APOA4, APOA1B, APOL1, APOL5, APOL6, APOB, APOO, FABP3, MSR1), lipid metabolism ( $L P L, C H 25 H)$, cell adhesion (ICAMI, VCAM1, CD9, VWF, SCARF1, SMOC2, ANTXR2), angiogenesis (VAV3, ESM1, ANGPT4, SERPINEF1), proteolysis (SERPINA9, PAPPA2, UBE2G1), endothelial cell cycle (THAP1), cytoskeleton function (TNNT2, TNNI3), Wnt signaling (AMOTL2, PPAP2B, TLE2), mTOR signaling (LAMTOR2, DDIT4L), endocytotic signaling (LRP8), apoptotic signalling (TNFRSF12A), phospholipid metabolism (PPAP2B, PPAP2C), blood clotting ( $P L G)$, oxygen transport $(M B)$, detoxification $(C Y P 2 S 1)$, extracellular matrix organization (HPSE2), purine biosynthesis (ADSSL1), nucleotide metabolism (NT5DC4), endoplasmic reticulum stress (HERPUD2), organic anion transport (SLC22A9), glucose transport (SLC2A12), lysosome function (CYBASC3), and immunity (antigen processingIFI30; protein ubiquitination-MARCH1; inhibition of IFNG release from T cells-BTN3A2).

Genes down-regulated by Hcy-thiolactone include those involved in chromatin modification/transcription regulation (H2AK119 ubiquitination $C B X 2$; methylationDOT1L, SUV42OH2, SMYD3; demethylation-JMJD2B), nucleosome assembly (HIST1H2AJ, HIST1H1B, BRD8), one-carbon metabolism (SHMT1), lipid metabolism/ transport (NCEH1, ANGPTL3, APOA5, HDLBP), angiogenesis (ANGPT2, APOLD1, VEGFA, MMP19, PTPRB), blood coagulation ( $F G B, A N X A 8, I R F 2)$, cell adhesion (PCDHB7, MMRN1), immunity/cell adhesion (CD209), energy metabolism/glycolysis $(P F K)$, cytokine signaling (IL20RA, ClQTNF4), signaling (PHACTR4, PTPN6), glycolipid biosynthesis (PIGL), cell morphology (ZNF135), protein ubiquitination (IBRDC2), protein deubiquitination (USP11), transmembrane transport (choline transporterSLC44A3), cytoskeleton assembly (KRT27), extracellular matrix organization (NTN4), endoplasmic reticulum quality control (OS9), N-glycan processing (ST8SIA2), and unknown function (CCDC129, LRRIQ1, SCUBE1). 
Table 3 List of $N$-Hcy-protein-responsive genes and their expression levels

\begin{tabular}{|c|c|c|c|c|}
\hline \multirow[t]{2}{*}{ Gene name } & \multirow{2}{*}{$\begin{array}{l}\text { Accession } \\
\text { number }\end{array}$} & \multirow[t]{2}{*}{ Protein name } & \multicolumn{2}{|l|}{ Rel. expression, fold } \\
\hline & & & $10 \mu \mathrm{M} N$-Hcy-protein & $40 \mu \mathrm{M} \mathrm{N}$-Hcy-protein \\
\hline$B C N P 1$ & 199,786 & Family with sequence similarity 129 , member C & -1.1 & -6.01 \\
\hline CGI-12 & 51,001 & MTERF domain containing 1 & -1.14 & -8.97 \\
\hline CHD5 & 1003 & Cadherin 5, type 2 (vascular endothelium) & 1.16 & 3.95 \\
\hline CORIN & 10,699 & Corin, serine peptidase & -6.36 & -21.04 \\
\hline$D D X 28$ & 55,794 & DEAD (Asp-Glu-Ala-Asp) box polypeptide 28 & -6.11 & -22.04 \\
\hline DNAJB7 & 150,353 & DnaJ (Hsp40) homolog, subfamily B, member 7 & 2.47 & 10.41 \\
\hline DOCK1 & 1793 & Dedicator of cytokinesis 1 & -8.02 & -15.11 \\
\hline$D P E P 2$ & 64,174 & Dipeptidase 2 & -1.39 & -22.66 \\
\hline$E D F 1$ & 8721 & Endothelial differentiation-related factor 1 & -1.98 & -2.25 \\
\hline EEF1A1 & 1915 & Eukaryotic translation elongation factor 1 alpha 1 & -2.5 & -46.91 \\
\hline EIF3S6IP & 51,386 & $\begin{array}{l}\text { Eukaryotic translation initiation factor } 3 \text {, subunit } 6 \text { interacting } \\
\text { protein }\end{array}$ & -1.02 & -8.88 \\
\hline EPHX1 & 2052 & Epoxide hydrolase 1, microsomal & 4.71 & 9.53 \\
\hline ESM1 & 11,082 & Endothelial cell-specific molecule 1 & 19.6 & 10.55 \\
\hline KRT25C & 147,183 & Keratin $25 \mathrm{C}$ & -3.86 & -4.06 \\
\hline LOC40139 & 4013 & Hypothetical gene supported by BC063892 & -1.1 & -4.54 \\
\hline$L R R I Q 1$ & 84,125 & Leucine-rich repeats and IQ motif containing 1 & -1.15 & -30.41 \\
\hline MELL1 & 27,390 & Mel transforming oncogene-like 1 & -1.75 & -48.02 \\
\hline$M P O$ & 4353 & Myeloperoxidase & -6.36 & -4.66 \\
\hline MRPS5 & 64,969 & Mitochondrial ribosomal protein S5 & 2.56 & 12.55 \\
\hline MYL6 & 4637 & Myosin, light-chain 6 & 1.68 & 7 \\
\hline$M Y L 12 B$ & 103,910 & Myosin, light-chain 12B, regulatory & 1.79 & 48.46 \\
\hline OS9 & 10,956 & Osteosarcoma amplified 9 , endoplasmic reticulum lectin & 2.41 & 21.88 \\
\hline PHACTR4 & 65,979 & Phosphatase and actin regulator 4 & -1.21 & -4.69 \\
\hline$P L B 1$ & 151,056 & Phospholipase B1 & 1.14 & 2.26 \\
\hline$P R K A B 2$ & 5565 & Protein kinase, AMP-activated, beta 2 non-catalytic subunit & -3.13 & -9.81 \\
\hline$R P 11-30 M 20.2$ & 26,121 & PRP31 pre-mRNA processing factor 31 homolog (S. cerevisiae) & 2.56 & 4.12 \\
\hline SLC25A18 & 83,733 & Solute carrier family 25 (glutamate carrier), member 18 & 4.99 & 8.67 \\
\hline$S P R Y 3$ & 10,251 & Sprouty homolog 3 (Drosophila) & -1.49 & -10.74 \\
\hline SULT1E1 & 6783 & Sulfotransferase family 1E, estrogen-preferring, member 1 & -1.7 & -9.26 \\
\hline ZNF227 & $1,357,694$ & Zinc finger protein 227 & -1.39 & -29.87 \\
\hline
\end{tabular}

It should be noted that in HUVEC cultures, Hcy-thiolactone turns over with a half-life of $3.5 \mathrm{~h}$ (Jakubowski et al. 2000). Thus, when $10 \mu \mathrm{M}$ Hcy-thiolactone is added to cell cultures, its concentration drops by 2-fold every $3.5 \mathrm{~h}$ and reaches $70 \mathrm{nM}$ at the end of a 24-h treatment. This range of Hcy-thiolactone concentrations corresponds to the values observed in human plasma from CBS-deficient patients and in mouse models of HHcy (Chwatko et al. 2007).

\section{Genes affected by $N$-Hcy-protein}

Treatment of HUVECs with $N$-Hcy-protein caused changes in the expression of forty-seven genes (Table 3). Significant changes in mRNA levels were observed when the cells were treated with $10.1 \mu \mathrm{M} N$-Hcy-protein and were enhanced at $40.1 \mu \mathrm{M} N$-Hcy-protein. Eleven mRNAs were up-regulated and nineteen were down-regulated by $N$-Hcyprotein. The up-regulation varied from 2.3-fold for the phospholipase B1 PLB1 gene to 48.5-fold for the myosin light-chain 12B MYL12B gene. The down-regulation was from -2.3 -fold for the endothelial differentiation-related factor $1 E D F 1$ gene to -46.9 -fold for the eukaryotic translation elongation factor 1 alpha $1 E E F 1 A 1$ gene.

Genes up-regulated by $N$-Hcy-protein include those involved in the regulation of cell morphology (MYL12B, $M Y L 6$ ), endoplasmic reticulum quality control (OS9), protein biosynthesis (MRPS5), angiogenesis (endothelial cellspecific molecule $1-E S M 1)$, protein folding (DNAJB7), mRNA splicing (PRP31), transcription regulation 
Table 4 List of Hcy-responsive-genes and their expression levels

\begin{tabular}{|c|c|c|c|c|}
\hline \multirow[t]{2}{*}{ Gene name } & \multirow[t]{2}{*}{ Accession number } & \multirow[t]{2}{*}{ Protein name } & \multicolumn{2}{|c|}{ Rel. expression, fold } \\
\hline & & & $10 \mu \mathrm{M} \mathrm{Hcy}$ & $1 \mathrm{mM} \mathrm{Hcy}$ \\
\hline$C D 209$ & 30,835 & CD209 molecule & -1.1 & -3.68 \\
\hline DNAJC2 & 129,450 & DnaJ homolog subfamily $\mathrm{C}$ member 28 & 1.18 & 3.99 \\
\hline IFI30 & 10,437 & Interferon gamma-inducible protein 30 & 1.83 & 3.71 \\
\hline$I G I P$ & 492,311 & IgA-inducing protein homolog (Bos taurus) & -1.93 & -3.09 \\
\hline KRT27 & 342,574 & Keratin 27 & -1.18 & -6.55 \\
\hline LAMTOR2 & 28,956 & Late endosomal/lysosomal adaptor, MAPK and MTOR activator 2 & 1.21 & 4.9 \\
\hline$L M X 1 A$ & 4009 & LIM homeobox transcription factor 1, alpha & 2.58 & 2.69 \\
\hline$L R R I Q 1$ & 84,125 & Leucine-rich repeats and IQ motif containing 1 & -2.22 & -116.1 \\
\hline$M A R C H 1$ & 55,016 & Membrane-associated ring finger (C3HC4) 1, E3 ubiquitin-protein ligase & 1.28 & 3.72 \\
\hline PCDHB7 & 56,129 & Protocadherin beta 7 & -1.35 & -7.17 \\
\hline PHACTR4 & 65,979 & Phosphatase and actin regulator 4 & -1.31 & -7.61 \\
\hline SLC22A9 & 114,571 & Solute carrier family 22 (organic anion transporter), member 9 & 1.48 & 7.14 \\
\hline$S L C 44 A 3$ & 126,969 & Solute carrier family 44 , member 3 & -2.68 & -12.47 \\
\hline ST8SIA2 & 8128 & ST8 alpha- $N$-acetyl-neuraminide alpha-2,8-sialyltransferase 2 & -1.42 & -10.56 \\
\hline
\end{tabular}

(ATP-dependent helicase-CHD5), detoxification (epoxide hydrolase 1-EPHX1), membrane transport (mitochondrial glutamate carrier 2-SLC25A18), and lipid metabolism (phospholipase B1-PLB1).

Genes down-regulated by $\mathrm{N}$-Hcy-protein include those involved in phagocytosis (DOCK1), proteolysis (MELL1), protein biosynthesis (EIF3S6IP, EEF1A1, BCNP1), peptide hormone processing (CORIN), transcription regulation (ZNF227, MTERFD1, EDF1), RNA processing or transport (DDX28), signal transduction (SPRY3, PHACTR4), cytoskeleton assembly (KRT25C/27), energy metabolism (PRKAB2), leukotriene metabolism (DPEP2), host defense system (MPO), sulfation (SULT1E1), $\mathrm{N}$-glycan processing (ST8SIA2), and unknown function (LOC4013, LRRIQ1).

\section{Genes affected by Hcy}

Of the 30 genes whose expression was changed in endothelial cells treated with Hcy, 17 were up-regulated and 13 were down-regulated (Fig. 1; Table 4). Genes upregulated by Hcy include those involved in transmembrane transport (organic anion transporter-SLC22A9), mTOR signaling (LAMTOR2), transcription activation (DNAJC2), immunity (antigen processing-IFI30; protein ubiquitination-MARCHI).

Genes down-regulated by Hcy include those involved in transmembrane transport (choline transporter-SLC44A3), transcription activation ( $L M X 1 A)$, immunity ( $\operatorname{Ig} \mathrm{A}$ secretion-IGIP, cell adhesion-CD209), cell adhesion (PCDHB7), neurogenesis/signal transduction (PHACTR4), $\mathrm{N}$-glycan processing (ST8SIA2), cytoskeleton assembly
(KRT27), endoplasmic reticulum quality control (OS9), and unknown function (LRRIQ1) (Table 4).

It should be noted that Hcy can be attached to proteins, mostly the conserved Cys34 of albumin, via disulfide bonds and form $S$-Hcy-protein (Hortin et al. 2006; Jakubowski 2000, 2002b; Sengupta et al. 2001). Thus, it is possible that some of the changes in HUVEC gene expression in response to the supplementation with Hcy can be caused by $S$-Hcy-protein.

\section{Validation of microarray analyses by RT-qPCR}

To validate microarray results, we selected 20 genes that showed at least 4-fold change in mRNA level in response to $N$-Hcy-protein, Hcy-thiolactone, or Hcy and examined their expression by RT-qPCR (Fig. 1). For each of the 20 examined genes, RT-qPCR confirmed the microarray mRNA expression results. Genes such as $A H C Y, A P O L 1, M T R$, MTRR, ESM1, CBS, CD9, LAMTOR2, and MARCH1 that were up-regulated by Hcy-thiolactone (Table 2); OS9 and $D D X 28$ that were up-regulated by $N$-Hcy-protein (Table 3); LAMTOR2 and ST8SIA2 that were up-regulated by Hcy (Table 4) in microarray experiments were also found to be up-regulated in RT-qPCR experiments (Fig. 1a). SCUBE1, NCEH1, MMP19, DDX28, and ST8SIA2 that were downregulated by Hcy-thiolactone in microarray experiments (Table 2) were also found to be down-regulated in RTqPCR experiments (Fig. 1b). Some genes that were divergently regulated by each metabolite in microarray experiments (APOA5, ANXA8, NTN4, VEGFA, OS9, LAMTOR2, SLC44A3; Tables 2, 3, 4) were also found to be divergently regulated in RT-qPCR experiments (Fig. 1c). 

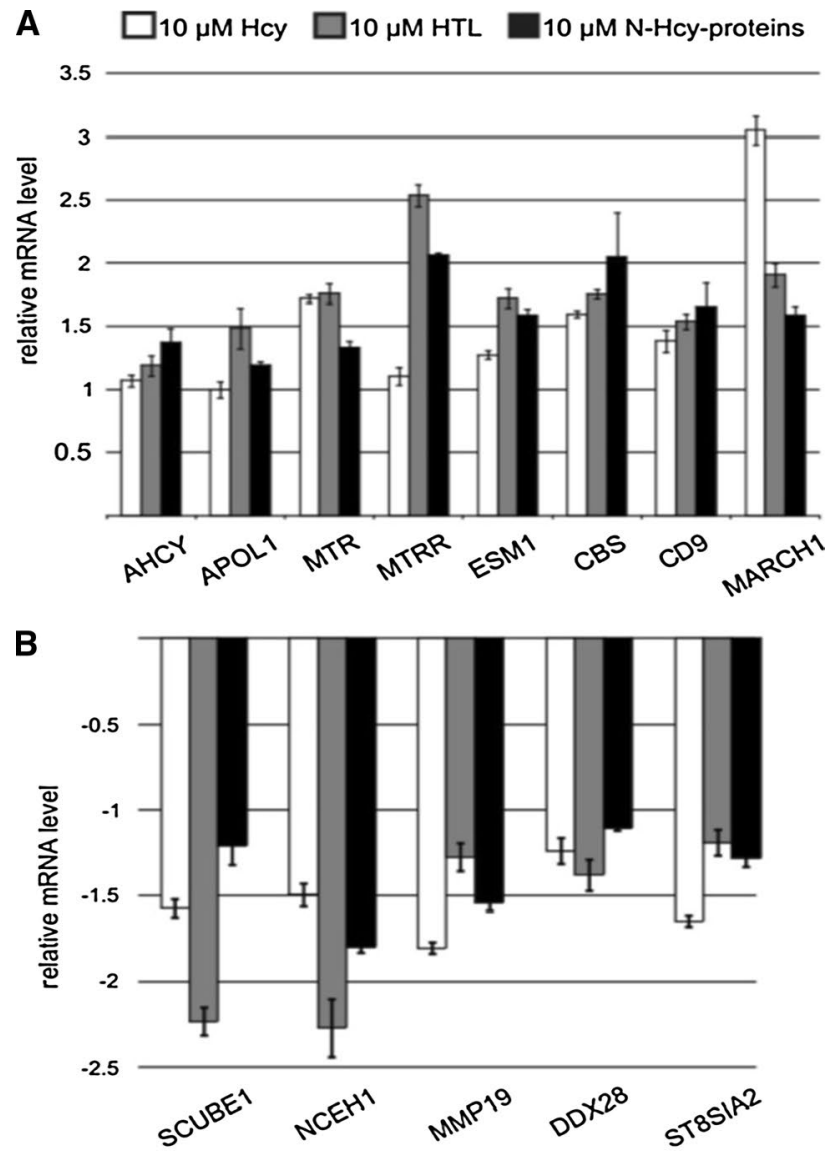

C

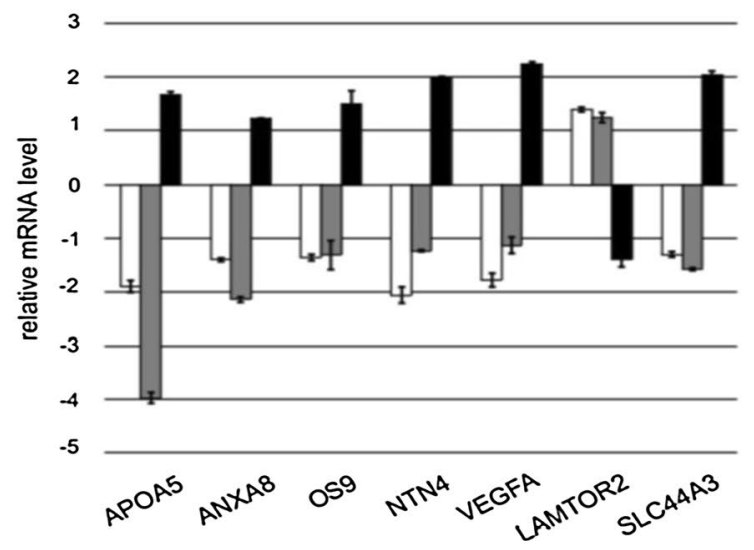

Fig. 1 Changes in gene expression induced by Hcy-thiolactone, $N$-Hcy-protein, and Hcy in human endothelial cells. mRNA levels were quantified by RT-qPCR, and values relative to untreated controls are shown. All changes are statistically significant $(P<0.05)$

\section{Bioinformatic analysis}

\section{Biological pathways and diseases}

To identify biological pathways and human diseases linked to genes responding to Hcy-thiolactone, $\mathrm{N}$-Hcy-protein, or Hcy, we carried out bioinformatic analysis using the PANTHER

\section{Hcy-thiolactone $\mathrm{N}$-Hcy-protein}

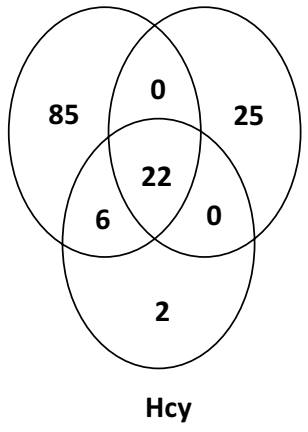

Fig. 2 Wenn diagram illustrating limited overlap between genes affected by Hcy-thiolactone, $N$-Hcy-protein, and Hcy

classification system and DAVID functional annotation tools. We found that genes affected by Hcy-thiolactone were significantly enriched in thirty molecular pathways, while genes affected by $\mathrm{N}$-Hcy-protein or Hcy were enriched in thirteen or eleven molecular pathways, respectively. Only five common pathways were enriched in genes affected by all three metabolites (Fig. 3a). Overall, there was very little overlap between molecular pathway categories enriched in genes affected by Hcy-thiolactone, $N$-Hcy-protein, or Hcy (Fig. 3a).

The majority of Hcy-thiolactone-affected pathway categories were not affected by $\mathrm{N}$-Hcy-protein or Hcy. Top five highly significant $[-\log (P$ value $)=20-31]$ pathway categories affected only by Hcy-thiolactone were 'chromatin organization,' 'one-carbon metabolism,' 'lipid localization,' 'lipoprotein metabolic processes,' and 'lipid and fatty acid transport' (Fig. 3a).

Most of the molecular pathway categories that were affected by $\mathrm{N}$-Hcy-protein or Hcy were also affected by Hcy-thiolactone. Five common pathways were enriched in genes affected by the three metabolites $[-\log (P$ value) $=4-11]$ : 'blood coagulation'; 'sulfur amino acid biosynthesis'; 'cysteine and methionine metabolism'; 'wound-healing'; and 'lipid, fatty acid, and steroid metabolism' [these pathways were also affected by Hcy-thiolactone, $-\log (P$ value $)=8-14]$ (Fig. 3a).

Six molecular pathway categories were enriched in genes affected only by $N$-Hcy-protein $[-\log (P$ value $)=4-7]$ : 'organic acid biosynthetic process,' 'focal adhesion,' 'biological oxidations,' 'endothelial cell differentiation,' 'epithelial cell differentiation,' 'plasma lipoprotein particle remodeling.' Only two molecular pathways were enriched in genes affected only by Hcy $[-\log (P$ value $)=4]$ : 'aspartate family amino acid metabolic process' and 'seleno amino acid metabolism.'

Eleven disease categories were found to be significantly related $[-\log (P$ value $)=4-16]$ to all three metabolites Hcy-thiolactone, $N$-Hcy-protein, and Hcy (Fig. 3b): 'down syndrome'; 'hyperhomocysteinemia'; 'cerebrovascular disease, ischemic disease'; 'atherosclerosis, coronary heart 
Table 5 List of genes whose expression was altered by all three metabolites

\begin{tabular}{|c|c|c|c|c|c|}
\hline \multirow[t]{2}{*}{ Gene name } & \multirow[t]{2}{*}{ Accession number } & \multirow[t]{2}{*}{ Protein name } & \multicolumn{3}{|c|}{ Change in expression induced by } \\
\hline & & & $N$-Hcy-protein & Hcy-thiolactone & Hcy \\
\hline$A H C Y$ & 191 & Adenosylhomocysteinase & $\uparrow$ & $\uparrow$ & $\uparrow$ \\
\hline ANXA8 & 653,145 & Annexin A8 & $\uparrow$ & $\downarrow$ & $\downarrow$ \\
\hline APOA5 & 116,519 & Apolipoprotein A-V & $\uparrow$ & $\downarrow$ & $\downarrow$ \\
\hline$A P O L 1$ & 8542 & Apolipoprotein L1 & $\uparrow$ & $\uparrow$ & \\
\hline$C B S$ & 875 & Cystathionine $\beta$-synthase & $\uparrow$ & $\uparrow$ & $\uparrow$ \\
\hline$C D 9$ & 928 & CD9 molecule & $\uparrow$ & $\uparrow$ & $\uparrow$ \\
\hline$D D X 28$ & 55,794 & DEAD (Asp-Glu-Ala-Asp) box polypeptide 28 & $\downarrow$ & $\downarrow$ & $\downarrow$ \\
\hline ESM1 & 11,082 & Endothelial cell-specific molecule 1 & $\uparrow$ & $\uparrow$ & $\uparrow$ \\
\hline LAMTOR 2 & 28,956 & Late endosomal/lysosomal adaptor, MAPK and MTOR activator 2 & $\downarrow$ & $\uparrow$ & $\uparrow$ \\
\hline LRRIQ1 & 84,125 & Leucine-rich repeats and IQ motif containing 1 & $\downarrow$ & $\downarrow$ & $\downarrow$ \\
\hline MMP19 & 4327 & Matrix metallopeptidase 19 & $\downarrow$ & $\downarrow$ & $\downarrow$ \\
\hline MTR & 4548 & 5-Methyltetrahydrofolate-homocysteine methyltransferase & $\uparrow$ & $\uparrow$ & $\uparrow$ \\
\hline MTRR & 4552 & 5-Methyltetrahydrofolate-homocysteine methyltransferase reductase & $\uparrow$ & $\uparrow$ & $\uparrow$ \\
\hline NCEH1 & 57,552 & Neutral cholesterol ester hydrolase 1 & $\downarrow$ & $\downarrow$ & $\downarrow$ \\
\hline NTN4 & 59,277 & Netrin 4 & $\uparrow$ & $\downarrow$ & $\downarrow$ \\
\hline OS9 & 10,956 & Osteosarcoma amplified 9, endoplasmic reticulum lectin & $\uparrow$ & $\downarrow$ & $\downarrow$ \\
\hline PHACTR4 & 65,979 & Phosphatase and actin regulator 4 & $\downarrow$ & $\downarrow$ & $\downarrow$ \\
\hline SCUBE1 & 80,274 & Signal peptide, CUB domain, EGF-like 1 & $\downarrow$ & $\downarrow$ & $\downarrow$ \\
\hline$S L C 44 A 3$ & 126,969 & Solute carrier family 44 , member 3 & $\uparrow$ & $\downarrow$ & $\downarrow$ \\
\hline ST8SIA2 & 8128 & ST8 Alpha- $N$-acetyl-neuraminide alpha-2,8-sialyltransferase 2 & $\downarrow$ & $\downarrow$ & $\downarrow$ \\
\hline$V E G F A$ & 7422 & Vascular endothelial growth factor A & $\uparrow$ & $\downarrow$ & $\downarrow$ \\
\hline
\end{tabular}

Up $(\uparrow)$ and down $(\downarrow)$ arrows indicate up-regulated and down-regulated gene expression

disease'; 'heart anomalies, congenital heart disease'; 'cancer'; 'non-Hodgkin lymphoma'; 'preterm delivery'; 'chromosomal damage'; 'neural tube defects'; and 'Alzheimer's disease.' One disease category 'cardiovascular disease' was significantly related to Hcy-thiolactone and $\mathrm{N}$-Hcy-protein $[-\log (P$ value $)=9-10]$.

Twelve disease categories were found to be significantly related $[-\log (P$ value $)=4-14]$ only to Hcy-thiolactone (Fig. 3b): 'lipid concentrations, risk of CAD'; 'polygenic dyslipidemia'; 'restenosis'; 'deep vein thrombosis'; 'stroke'; 'myocardial infarction'; 'aging'; 'venous thromboembolism'; 'placental abruption'; 'inflammatory bowel disease'; 'albuminuria'; 'cardiac hypertrophy'; and 'arterial hypertension.'

Seven disease categories were significantly $[-\log (P$ value $=4-8]$ related only to $N$-Hcy-protein: 'metabolic disease,' 'developmental diseases,' 'endometriosis,' 'albuminuria,' 'arterial hypertension,' 'DNA adducts,' and 'neurological diseases.' However, none of these disease categories was associated only with Hcy (Fig. 3b).

\section{Network analysis}

IPA identified a unique set of biological networks for each metabolite: eight for Hcy-thiolactone, three for
$N$-Hcy-protein, and another three for Hcy. Two topscored networks for Hcy-thiolactone (score = 34-40) were 'cardiovascular disease, cardiac infarction, skeletal and muscular system development and function' and 'cardiovascular disease, lipid metabolism, small molecule biochemistry' (Table 6). Two top-scored networks for $N$-Hcy-protein (score $=24-35$ ) were 'small molecule biochemistry, neurological disease' and 'cardiovascular system development and function' (Table 6). Two topscored networks for Hcy ( acid metabolism, lipid metabolism,' 'cellular movement, cardiovascular and nervous system development and function' (Table 6).

A predominant network, with a score $=40$, associated with Hcy-thiolactone contained 34 genes: 20 were quantified by microarrays and 14 were identified by IPA to interact with the quantified genes. Hcy-thiolactone exhibited strong interactions with histone/chromatin modification and transcription genes, endothelial cell growth, lipoprotein-related, and blood clotting genes (Fig. 4).

Different networks were associated with $\mathrm{N}$-Hcy-protein and Hcy. The predominant $N$-Hcy-protein network, with a score $=35$, contained 35 genes: 16 were identified by 

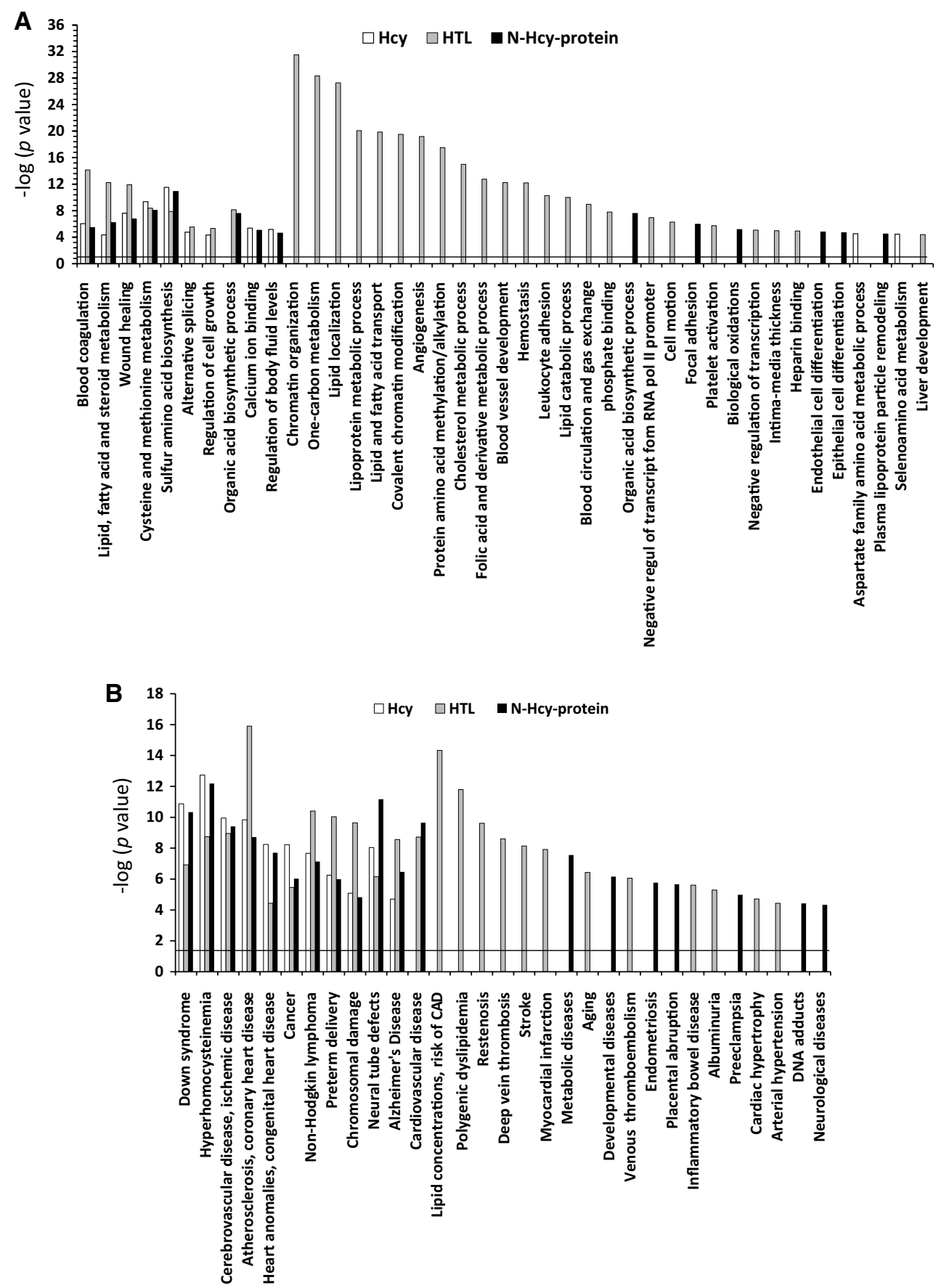

Fig. 3 Molecular pathways and disease associated with Hcy-thiolactone, $N$-Hcy-protein, and Hcy identified by DAVID tool. The analysis utilized $P$ value 0.05 , and Benjamini-Hochberg, Bonferroni, and

microarrays and 19 were identified by IPA to interact with the quantified genes (Fig. 5). This network reveals strong interaction of $\mathrm{N}$-Hcy-protein with genes involved in cardiovascular system development and function.

The predominant Hcy network, with a score $=37$, contained 35 genes: 16 were quantified by microarrays
FDR corrections were applied for minimizing the number of false positives. Panel A, molecular pathways; Panel B, diseases

and 19 were identified by IPA to interact with the quantified genes. The IPA-identified network for genes regulated by Hcy revealed strong interactions of those genes with the ubiquitin $\mathrm{C}$ (UBC) posttranslational modification system that targets proteins for proteolytic degradation (Fig. 6). 
Table 6 Top molecular networks of genes responding to Hcy-thiolactone, $N$-Hcy-protein, and Hcy

\begin{tabular}{|c|c|c|c|c|}
\hline Analysis & Molecules in Network & Score & \begin{tabular}{|c|} 
Focus \\
Molecules \\
\end{tabular} & $\begin{array}{c}\text { Top Diseases and } \\
\text { Functions } \\
\end{array}$ \\
\hline $\begin{array}{c}\text { Hcy- } \\
\text { thiolactone } \\
\text { (Fig. } 4 A)\end{array}$ & $\begin{array}{l}\text { 26s Proteasome, ATPase, Calcineurin protein(s), CBX2, Cyclin } \\
\text { A, DOT1L, E2f, EHMT1, EHMT2, EP300, EZH2, HIST1H1B, HISTONE, } \\
\text { Histone h3, histone-lysine N-methyltransferase, Hsp70, } \\
\text { Hsp90, JARID2, KDM4B, MBD3, MED26, Myosin, NFkB } \\
\text { (complex), Notch, NSD1, RNA polymerase II, SETD2, SETD7, } \\
\text { SLC2A12, SMOC2, SMYD3, TNNI3, TNNT2, Ubiquitin, USP11 }\end{array}$ & 40 & 20 & $\begin{array}{l}\text { Cardiovascular } \\
\text { Disease, Cardiac } \\
\text { Infarction, Skeletal } \\
\text { and Muscular } \\
\text { System Develop- } \\
\text { ment and Function }\end{array}$ \\
\hline $\begin{array}{c}\text { Hcy- } \\
\text { thiolactone } \\
\text { (Fig. 4B) }\end{array}$ & $\begin{array}{l}\text { Akt, ANGPT4,ANGPTL3, Ap1, APOA4, APOA5, APOB, APOL1, } \\
\text { BRD8, ELP2, EPC1, Growth hormone, H2AFY, HDL, HDL- } \\
\text { cholesterol, HIST1H2BJ/HIST1H2BK, Histone h4, IFN Beta, } \\
\text { IL12 (complex), IRF2, LDL, LDL-cholesterol, LPL, LRP, LRP8, } \\
\text { MAT1A, MSR1, NADPH oxidase, NCOR-LXR-Oxysterol-RXR-9 } \\
\text { cis RA, NfkB1-RelA, Pro-inflammatory Cytokine, SUV420H2, } \\
\text { VCAM1, VLDL, VLDL-cholesterol }\end{array}$ & 34 & 18 & $\begin{array}{l}\text { Cardiovascular } \\
\text { Disease, Lipid } \\
\text { Metabolism, Small } \\
\text { Molecule } \\
\text { Biochemistry }\end{array}$ \\
\hline $\begin{array}{l}\mathrm{N} \text {-Hcy- } \\
\text { protein } \\
\text { (Fig.5A) }\end{array}$ & $\begin{array}{l}\text { BAIAP2L1, CERCAM, CHD4, CHD5, COLGALT1, DERL2, DPEP2, } \\
\text { EIF3L, EPHX1, EPM2A, ERLEC1, FOXRED2, GSK3B, GYG1, } \\
\text { KCTD5, LAMTOR2, MAP3K6, MAP3K15, MARCH1, MARCH8, } \\
\text { MRPS5, MYL6, MYL12A,NCEH1, NHLRC1, NUP210, OS9, PRKAB2, } \\
\text { PRKAG2, SLC25A18, SLC44A3, STIM2, UBC, Zfp819, ZNF227 }\end{array}$ & 35 & 15 & $\begin{array}{l}\text { Small Molecule } \\
\text { Biochemistry, } \\
\text { Neurological } \\
\text { Disease }\end{array}$ \\
\hline $\begin{array}{l}\mathrm{N} \text {-Hcy- } \\
\text { protein } \\
\text { (Fig.5B) }\end{array}$ & $\begin{array}{l}\text { 16,16-dimethylprostaglandin E2, acetic acid, AHCY, Akt, } \\
\text { APOA5, APOL1, CD9, Cg, CRK/CRKL, DOCK1, Dock1-Pak, } \\
\text { EDF1, EEF1A1, ELMO1, ERK1/2, ESM1, Focal adhesion kinase, } \\
\text { GPIHBP1, HDL, Insulin, LDL, MMP19, MPO, nicotinic acid, } \\
\text { NTN4, PI3K (complex), Pkc(s), PLB1, Rac, SCUBE1, Sos, SRC } \\
\text { (family), SULT1E1, VEGFA, ZHX2 }\end{array}$ & 35 & 15 & $\begin{array}{l}\text { Cardiovascular } \\
\text { System Develop- } \\
\text { ment and Function, } \\
\text { Dermatological } \\
\text { Diseases and } \\
\text { Conditions }\end{array}$ \\
\hline $\begin{array}{c}\text { Hcy } \\
\text { (Fig, 6A) }\end{array}$ & $\begin{array}{l}\text { ACOX1, AHCY, ANXA8L2(includes others), ATP5G3, ATP8B2, } \\
\text { ATP9A, CERCAM, COLGALT1, CSTB,DNAJC2, DPP9, FOXRED2, } \\
\text { GSTP1, HLA-DOA, HLA-DRB1, IFI30, INSR, KRT27, LAMTOR2, LDB2, } \\
\text { LYVE1, MARCH1, MARCH8, MTR, MTRR, MYL6, OS9, PCDHB7, } \\
\text { RDH12, SLC22A9, SLC44A3, SMTN, ST8SIA2, TGFB1, UBC }\end{array}$ & 37 & 15 & $\begin{array}{l}\text { Amino Acid } \\
\text { Metabolism, Lipid } \\
\text { Metabolism }\end{array}$ \\
\hline $\begin{array}{c}\text { Hcy } \\
\text { (Fig.6B) }\end{array}$ & $\begin{array}{l}\text { Akt, } \alpha \text {-estradiol, APOA5, CD9, Cg, cholesterol, CYB5B, ERK1/2, } \\
\text { ESM1, FKHR, Focal adhesion kinase, GPIHBP, HIF3A, HS6ST1, } \\
\text { HTATIP2, LMX1A, MMP15, MMP19, NCEH1, NDST1, NTN4, } \\
\text { PDGFD, PI3K (complex), PLXND1, PROK2, SCUBE1, SCUBE3, } \\
\text { SEMA3F, SLC12A6, SLIT2, SULT1E1, VEGFA,WISP3, WNT1 }\end{array}$ & 22 & 10 & $\begin{array}{l}\text { Cellular Movement, } \\
\text { Cardiovascular Sys- } \\
\text { tem Development } \\
\text { \& Function, Nervo- } \\
\text { us System Develop- } \\
\text { ment \& Function }\end{array}$ \\
\hline
\end{tabular}

Upregulated and downregulated genes are highlighter in red and green, respectively, Graphical representation of interactions between molecules in these networks is shown in Figs. 4, 5, and 6

\section{Discussion}

Endothelial dysfunction plays a central role in CVD and is a common finding in HHcy in humans and animal models (Dayal and Lentz 2008; Lentz 2005). To understand the mechanisms by which HHcy disrupts normal cellular function and ultimately causes disease, we studied human endothelial cell transcriptome to identify genes whose expression is modulated by individual Hcy metabolites that accumulate in HHcy. We examined changes in gene expression induced by Hcy-thiolactone, $\mathrm{N}$-Hcy-protein, and Hcy itself in HUVECs, a frequently used model of vascular cells (Jakubowski et al. 2000). We found that each metabolite affected the expression of unique sets of genes in different metabolic pathways and networks that are associated with CVD. Many of those genes and pathways were not previously associated with HHcy. Our results demonstrate that Hcy-thiolactone and $\mathrm{N}$-Hcy-protein, rather than Hcy, cause changes in gene expression for the majority of genes.

\section{Pathways affected by Hcy-thiolactone}

'Chromatin organization' was the top molecular pathway affected by Hcy-thiolactone (Fig. 3a). Out of 113 genes affected by Hcy-thiolactone, nineteen encoded chromatin proteins: histones, histone-modifying enzymes, and chromatin-binding proteins. These include histone-lysine methyltransferase genes SEDT2, SEDT7, EZH2, EHMT1, EHMT2, which were up-regulated, and $S U V 420 H 2$, DOTIL, SMYD3, which were down-regulated. Histonelysine acetyltransferase $(E P C 1, E P 300)$ and ubiquitin transferase $(C B X 2)$ genes were up-regulated, while methylCpG-binding domain protein $(M B D 3)$ and lysine-specific demethylase (JMJD2B) genes were down-regulated. Another gene, encoding regulator of histone methylation protein Jumonji (JARID2), was up-regulated by Hcy-thiolactone. These findings suggest that epigenetic mechanisms involving histone modification might contribute to endothelial dysfunction induced by Hcy-thiolactone. 
Fig. 4 Top networks of Hcythiolactone-responsive genes. a Cardiovascular disease, cardiac infarction, skeletal and muscular system development and function network; b cardiovascular disease, lipid metabolism, small molecule biochemistry network. Direct (solid lines) and indirect (dashed lines) genegene interactions between genes up-regulated ( $r e d)$, down-regulated (green), and unaffected (white) by Hcy-thiolactone. The intensity of green and red node colors indicates the degree of down- or up-regulation, respectively. Solid lines indicate simple binding associations between protein products of gene expression, whereas arrows indicate that the gene at the arrowhead is being acted upon by the gene at the origin of the arrow
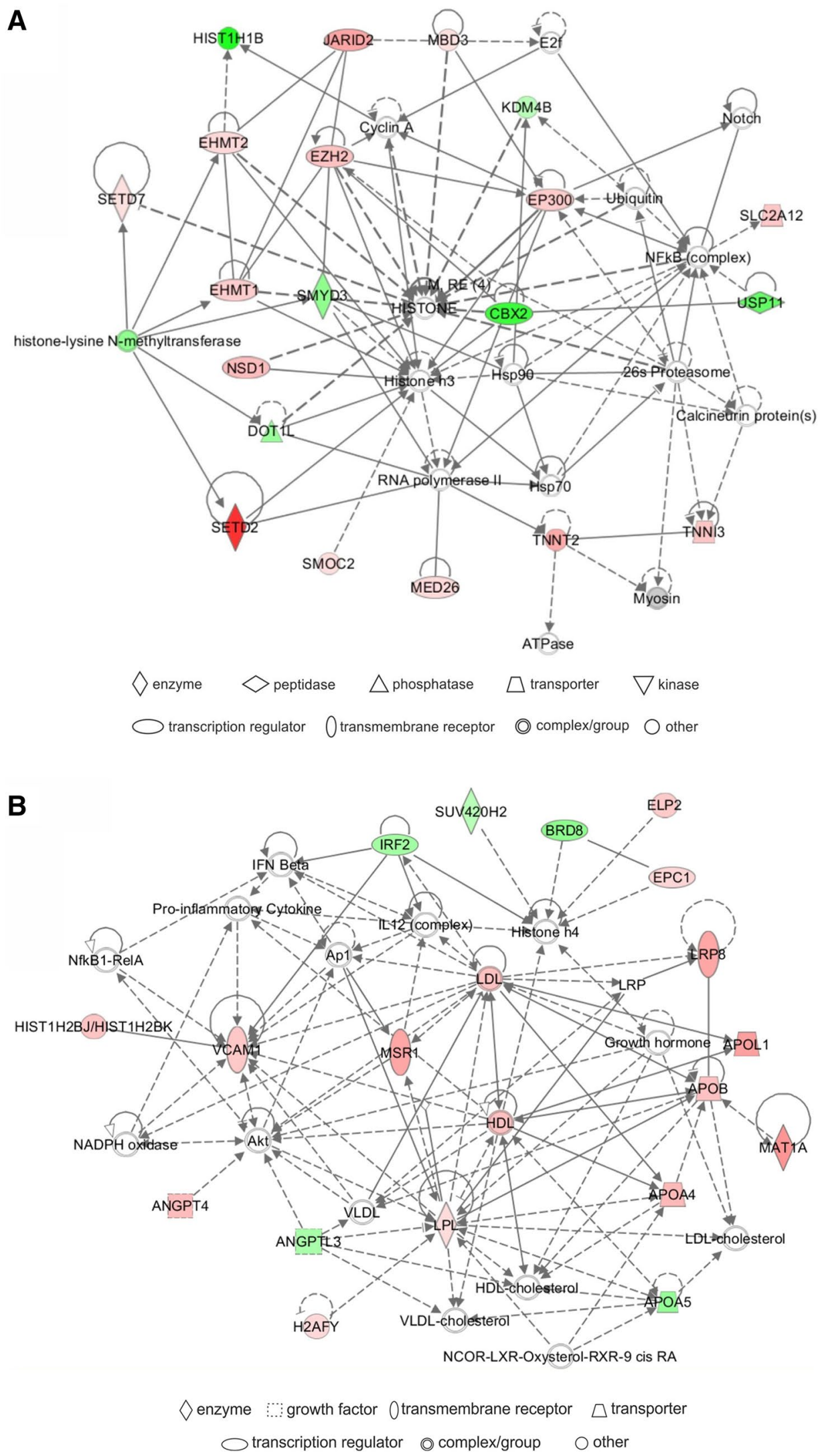
Fig. 5 Top networks of $N$-Hcyprotein-responsive genes. a Small molecule biochemistry, neurological disease network; $\mathbf{b}$ cardiovascular system development and function, dermatological diseases and conditions network. Direct (solid lines) and indirect (dashed lines) genegene interactions between genes up-regulated (red), down-regulated (green), and unaffected (white) by $\mathrm{N}$-Hcy-protein
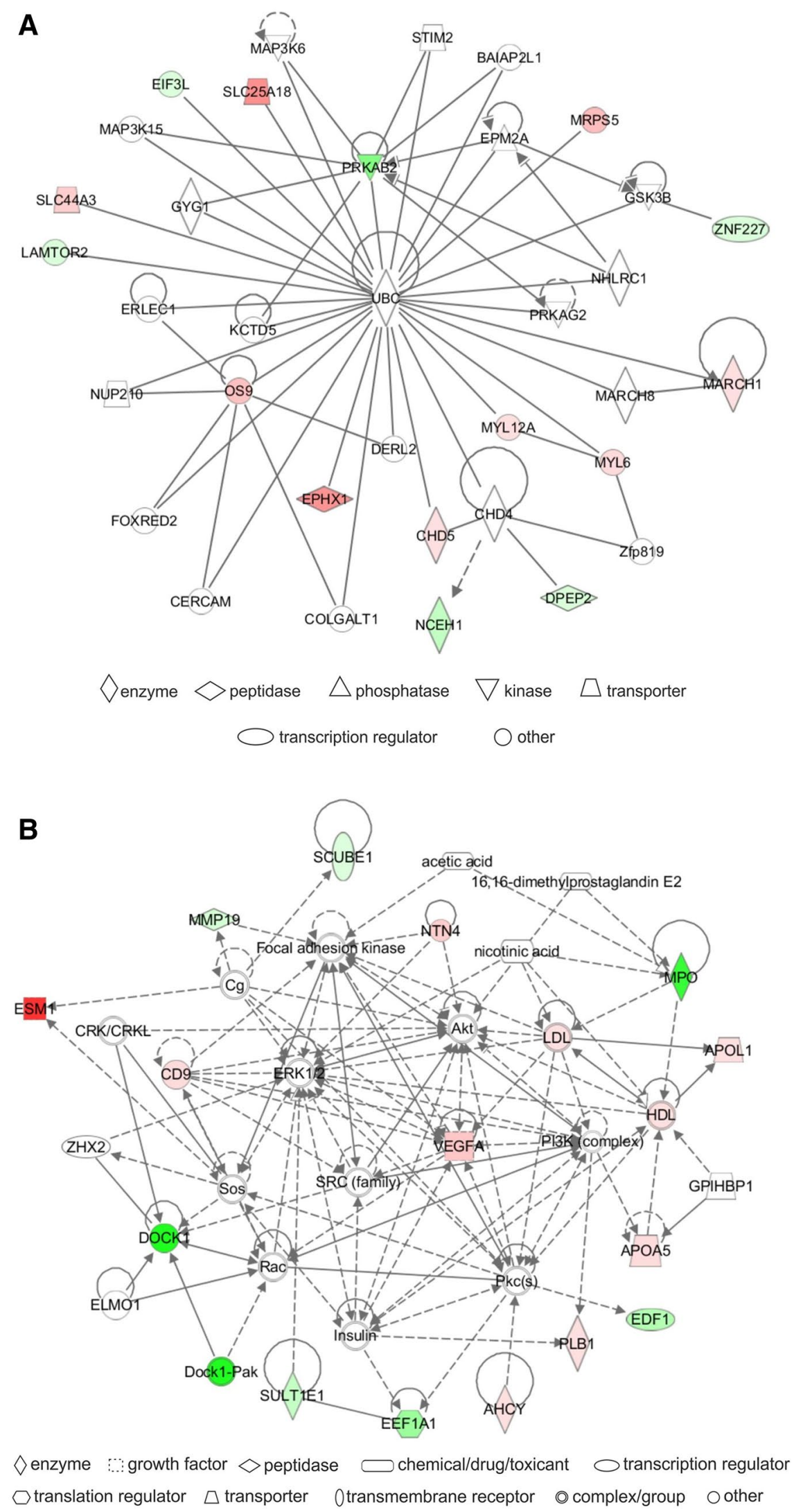
Fig. 6 Top networks of Hcyresponsive genes. a Amino acid metabolism and lipid metabolism network; b cellular movement, cardiovascular system development and function, and nervous system development and function network. Direct (solid lines) and indirect (dashed lines) gene-gene interactions between genes $u p$ regulated (red), down-regulated (green), and unaffected (white) by Hcy
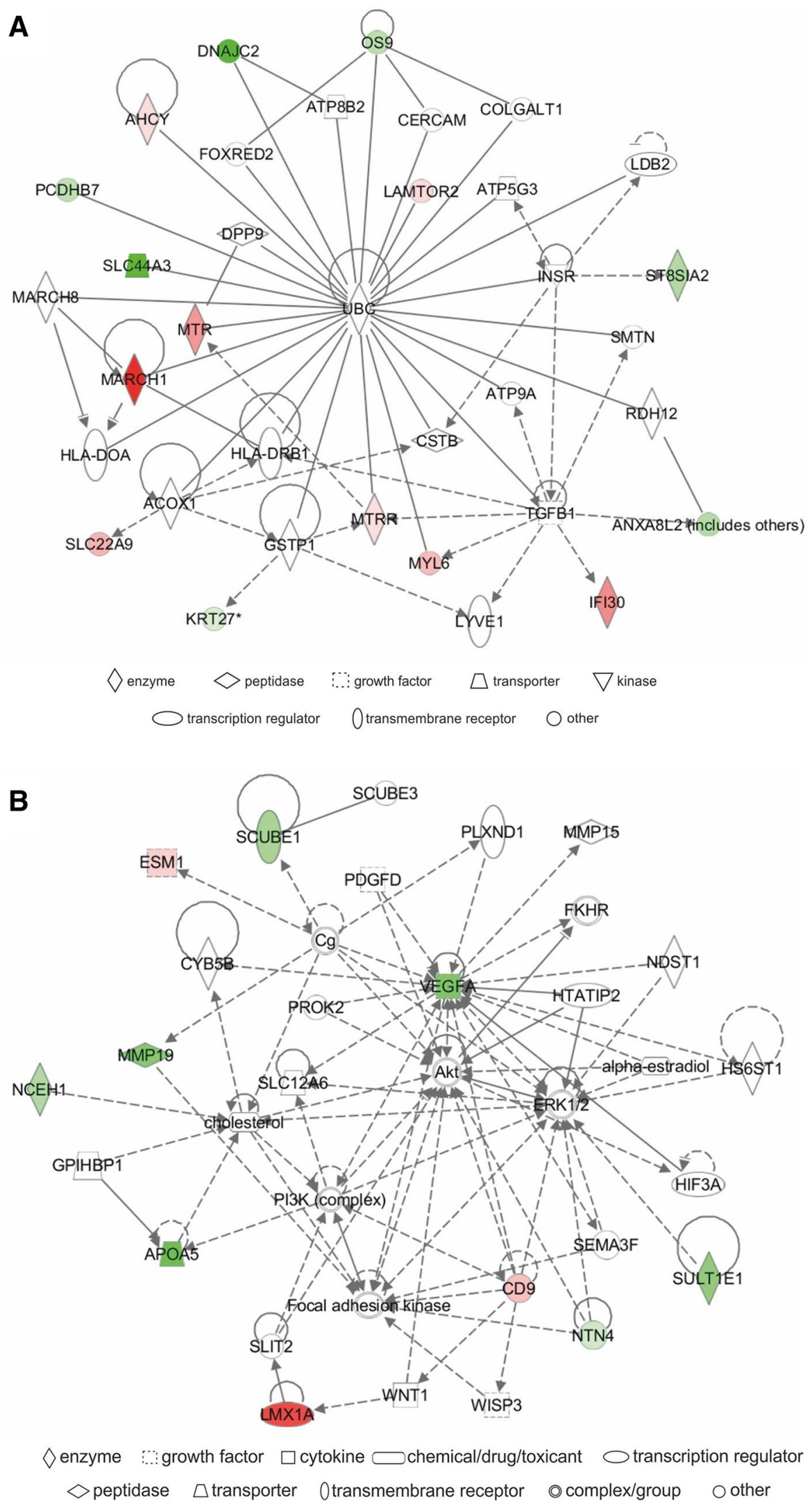
Posttranslational modifications of histone-lysine $\varepsilon$-amino groups by acetylation, methylation, and ubiquitination play a key role in the modulation of chromatin structure and DNA methylation status that govern epigenetic regulation of gene expression (Tessarz and Kouzarides 2014). Previous findings have shown that the status of DNA methylation can be affected by HHcy and that AdoHcy is responsible for epigenetic dysregulation of normal DNA methylation patterns associated with atherosclerosis (Handy et al. 2011; Ingrosso et al. 2003). However, other studies found that treatments with Hcy increase DNA methylation in human lymphocytes (Yi-Deng et al. 2007) and cardiomyocytes (Chaturvedi et al. 2014), suggesting that DNA methyltransferases are not inhibited by AdoHcy in those cells.

In mice, HHcy cause tissue-specific changes in H19 gene methylation and expression. In response to HHcy, H19 DNA methylation decreased in the liver but increased in the brain and aorta (Devlin et al. 2005). Furthermore, increased H19 DNA methylation resulted in a decrease in H19 expression in the brain but an increase in H19 expression was observed in the aorta. Levels of H19 transcripts in aorta correlated positively with plasma total Hcy (Devlin et al. 2005). These findings indicate that, in contrast to original suggestions (Castro et al. 2005; Jamaluddin et al. 2007), AdoHcy level may not be a major determinant of DNA methylation in HHcy and that other factors regulate DNA methylation and gene expression. Our present findings identify Hcy-thiolactone as a factor that can lead to epigenetic dysregulation of gene expression by affecting histones, histone-modifying enzymes, and other chromatinassociated proteins that control DNA methylation.

The involvement of a histone deacetylase and a methylCpG-binding protein, whose genes we found to be affected by Hcy-thiolactone, in the epigenetic modulation of gene expression by HHcy in vivo was documented in a mouse model in which HHcy was induced by subcutaneous administration of methionine $(5.2 \mathrm{mmol} / \mathrm{kg}$ twice a day, for 7 days) (Dong et al. 2007). This treatment down-regulates reelin (RELN) expression in GABAergic interneurons by inducing hypermethylation of $\mathrm{CpG}$ islands in the RELN promoter in mouse brain. This process involves recruitment of transcription repressors methyl-CpG-binding protein (MeCP2) and histone deacetylases (HDAC) leading to the formation of transcriptionally inactive chromatin. Histone hyperacetylation induced by the administration of HDAC inhibitors leads to demethylation and activation of RELN promoter. DNA methyltransferase DNMT1, whose expression is not changed by Met treatment, is not responsible for RELN promoter demethylation (Dong et al. 2007).

'One-carbon metabolism' was the second top pathway affected by Hcy-thiolactone (Fig. 3a). We found that MTHFD2 gene encoding mitochondrial bifunctional methylenetetrahydrofolate dehydrogenase/cyclohydrolase was up-regulated in HUVECs treated with Hcy-thiolactone, but not Hcy or $\mathrm{N}$-Hcy-protein. Other investigators reported that MTHFD2 is up-regulated in HUVECs treated with $10 \mathrm{mM}$ Hcy, but not in cells treated with $1 \mathrm{mM}$ Hcy (Kokame et al. 1996). However, because treatments of HUVECs with such high Hcy concentrations generate significant concentrations of Hcy-thiolactone in a reaction catalyzed by methionyl-tRNA synthetase (even in the presence of competing methionine) (Jakubowski 2002a; Jakubowski et al. 2000), our present results strongly suggest that the actual metabolite inducing MTHFD2 expression in the Kokame et al.'s experiments was Hcy-thiolactone rather than Hcy.

Three other genes of the 'one-carbon metabolism' pathway, $C B S, M T R$, and MTRR, that we found to be upregulated by Hcy-thiolactone, $N$-Hcy-protein, and Hcy are known to be associated with CVD (Zhao et al. 2012). ALDH1L1, another gene of the 'one-carbon metabolism' pathway that we found to be up-regulated by Hcy-thiolactone, is associated with ischemic stroke (Williams et al. 2014). Since reduced activity of these genes causes disease, our findings suggest that their up-regulation induced by Hcy metabolites will facilitate Hcy metabolism and thus is protective. This conclusion is supported by findings from a genome-wide meta-analysis showing that $A L D H 1 L 1$ and $C B S$ are strongly associated with the difference between pre- and post-methionine load test total Hcy levels (Williams et al. 2014).

Other top pathways affected by Hcy-thiolactone were related to lipid traits. Serum total cholesterol, low-density lipoprotein cholesterol, high-density lipoprotein cholesterol, and triglycerides are among the most important risk factors for CVD and are targets for therapeutic intervention. Of the several genes involved in 'lipid localization,' 'lipoprotein metabolic processes,' and 'lipid and fatty acid transport' pathways whose expression was affected by Hcythiolactone, four (ANGPTL3, APOA1B, APOB, LPL) are known to be associated with lipid traits (Teslovich et al. 2010). These findings are consistent with previous results showing that lipid metabolism is deregulated in HHcy (Maclean et al. 2012; Werstuck et al. 2001) and suggest that Hcy-thiolactone contributes to these disturbances of lipid homeostasis. We also found that macrophage scavenger receptor gene (MSRl) implicated in cholesterol deposition in arterial walls during atherogenesis (Gough et al. 1998) was up-regulated by Hcy-thiolactone. This finding suggests a possible mechanistic link between HHcy and atherogenesis.

We also found that two genes encoding protein components of the mTOR signalling pathway were up-regulated by Hcy-thiolactone: the ragulator complex protein LAMTOR2 that recruits mTORC1 to lysosomes (Nada 
et al. 2009) and the DNA-damage-inducible transcript 4-like protein DDIT4L, a negative regulator of signal transduction that inhibits cell growth by regulating the TOR signaling pathway upstream of the TSC1-TSC2 complex (Corradetti et al. 2005). Amino acid sensing by mTORC1 involves leucyl-tRNA synthetase (Han et al. 2012), which is also known to metabolize Hcy to Hcy-thiolactone (Jakubowski 1995, 2011, 2012, 2013; Sikora and Jakubowski 2009). Our findings that LAMTOR2 expression is down-regulated by $\mathrm{N}$-Hcy-protein and up-regulated by Hcy (Fig. 1c) highlight the unique role of each Hcy metabolite in mTOR signalling. Up-regulation of DDIT4L that we observe in endothelial cells in response to Hcythiolactone is also observed in atherosclerotic lesions relative to healthy segments of the same artery (Cuaz-Perolin et al. 2004). Since protein $N$-homocysteinylation by Hcythiolactone occurs in atherosclerotic lesions, as shown in ApoE-/- mouse model (Perla-Kajan et al. 2008), these findings provide further support for a pro-atherogenic role of Hcy-thiolactone.

\section{Pathways affected by Hcy-thiolactone, $N$-Hcy-protein, and Hcy}

Our finding that the blood coagulation pathway is affected by Hcy-thiolactone, $\mathrm{N}$-Hcy-protein, and Hcy in a metabolite-specific manner is consistent with a pro-thrombotic phenotype of HHcy patients and suggests that each metabolite contributes to thrombosis. Severe HHcy is associated with thromboembolism, the major cause of morbidity and mortality in homocystinuric patients (Mudd et al. 1985). Mild HHcy is also a risk factor for venous thrombosis in the general population (Den Heijer et al. 2005). Previous findings demonstrated the involvement of Hcy-thiolactone and Hcy in two distinct mechanisms that may contribute to pro-thrombotic phenotype associated with HHcy. One mechanism involves the modification of fibrinogen (Fbg) by Hcy-thiolactone, which generates $\mathrm{N}$-Hcy-Fbg that forms fibrin clots resistant to lysis by plasmin (Sauls et al. 2006). Hcy-thiolactone and $N$-Hcy-protein, including pro-thrombotic $N$-Hcy-Fbg (Sauls et al. 2006, 2011), are elevated in homocystinuric patients (Jakubowski et al. 2008). One of the three $\mathrm{N}$-Hcy-lysine residues in Fbg from homocystinuric patients, $\mathrm{N}$-Hcy-Lys562, is located in an unstructured region ( $\alpha 392-610)$ of the $\alpha$-chain known to be involved in IPA and plasminogen binding, which can explain the abnormal characteristics of fibrin clots formed from $N$-Hcy-Fbg (Jakubowski 2013; Sikora et al. 2014). In another mechanism, based on studies in mice with dietary HHcy, Hcy was shown to form a disulfide linkage with Cys9 residue of annexin $\mathrm{A} 2$, which reduces perivascular thrombolytic activity by preventing the cell surfacedependent plasmin generation (Jacovina et al. 2009).
Our present findings showing that the blood coagulation pathway is affected by Hcy-thiolactone, $\mathrm{N}$-Hcy-protein, and Hcy suggest that, in addition to direct effects on the components of blood clotting system such as Fbg and annexin A2, Hcy-thiolactone and Hcy may affect vascular homeostasis by changing the expression of genes encoding proteins involved in blood clotting (CD9, ANXA8, SCUBE1 for Hcy-thiolactone and Hcy; $V W F, F G B, M M R N 1, P L G$, $L R P 8$ for Hcy-thiolactone). Furthermore, some blood coagulation genes (CD9, ANXA8, SCUBE1) may also be affected by $N$-Hcy-protein. Taken together, these findings suggest that each of the three metabolites exerts a specific effect on vascular homeostasis. In addition, elevated expression of VWF in response to Hcy-thiolactone may contribute to increased restenosis observed in cardiac surgery patients with HHcy (Schnyder et al. 2001).

We found that MTR, MTRR, AHCY, and CBS genes encoding enzymes involved in sulfur amino acid metabolism were up-regulated by Hcy-thiolactone, $N$-Hcy-protein, and Hcy. Of these, low activity CBS, MTR, and MTRR variants are known to be associated with CVD (Zhao et al. 2012). These findings suggest a protective mechanism in which the accumulation of toxic Hcy metabolites such as Hcy-thiolactone and $\mathrm{N}$-Hcy-protein up-regulates the expression of genes involved in the removal of Hcy via trans-methylation and trans-sulfuration pathways that prevent the accumulation of these toxic metabolites. That CBS is important for maintaining endothelial cell homeostasis is supported by findings showing that reduction in CBS expression induces premature senescence in HUVECs (Albertini et al. 2012).

Highly significant molecular pathways affected by Hcythiolactone, $\mathrm{N}$-Hcy-protein, and Hcy include the 'lipid, fatty acid, and steroid metabolism' pathway. Fifteen genes of this pathway were affected by Hcy-thiolactone, seven genes were affected by $N$-Hcy-protein, and four by Hcy. Of these, three genes (ANXA8, APOA5, ST8SIA2) were affected by each of the three metabolites, and one gene (SULTIE1) was affected by $N$-Hcy-protein and Hcy. These findings are consistent with lipid traits associated with HHcy (Maclean et al. 2012; Werstuck et al. 2001) and suggest that each of the three metabolites-Hcy-thiolactone, $\mathrm{N}$-Hcy-protein, and Hcy-may contribute to the modification of lipid traits in a metabolite-specific manner.

\section{Diseases related to Hcy-thiolactone, $N$-Hcy-protein, and Hcy}

Bioinformatic analyses show that most of the disease categories related to Hcy-thiolactone, $\mathrm{N}$-Hcy-protein, and Hcy involve the cardiovascular and central nervous systems. These diseases are known from previous studies to be associated with HHcy. However, our findings indicate 
that a specific Hcy metabolite is associated with a specific disease. Surprisingly, the 'cardiovascular disease' category was associated only with Hcy-thiolactone and $\mathrm{N}$-Hcy-protein, but not with Hcy. Furthermore, while some diseases were associated only with Hcy-thiolactone or $\mathrm{N}$-Hcy-protein, none was associated only with Hcy. Instead, diseases associated with Hcy were also associated with Hcy-thiolactone and $\mathrm{N}$-Hcy-protein. For example, we found that Hcythiolactone was related to coronary artery disease, dyslipidemia, deep vein thrombosis, stroke, myocardial infarction, venous thromboembolism, placental abruption, preeclampsia, cardiac hypertrophy, and neurological disease. These findings are consistent with clinical manifestations of severe HHcy in genetic deficiencies of Hcy metabolism (Mudd et al. 2001; Strauss et al. 2007) and in mild HHcy in the general population (Joseph and Loscalzo 2013; Refsum et al. 2006) and suggest that Hcy-thiolactone might play a predominant role in these diseases. Another metabolite, $\mathrm{N}$-Hcy-protein, might play a predominant role in developmental and neurological diseases.

We have also found that Hcy-thiolactone and $\mathrm{N}$-Hcyprotein, in addition to Hcy, are linked to other diseases including cerebrovascular disease, ischemic disease, congenital heart disease, Alzheimer's disease, and neural tube defects. Taken together, these findings suggest that each Hcy metabolite might have a specific disease-related role.

Our findings suggest that cancer is related to Hcy-thiolactone, $\mathrm{N}$-Hcy-protein, and Hcy (Fig. 3b). Previous studies have associated cancer with HHcy (Plazar and Jurdana 2010), and our present findings suggest that each of the three metabolites can contribute. Hcy metabolism is known to be deregulated in cancer cells, which in contrast to normal cells cannot grow on Hcy-containing media (Fiskerstrand et al. 1994). Hcy-thiolactone and $\mathrm{N}$-Hcy protein are elevated in cancer cells, which likely contributes to the growth defect on Hcy media (Jakubowski 1997a, b; Jakubowski and Goldman 1993). Deregulation of central carbon metabolic pathways has long been linked to cancer as discussed in a recent hypothesis (Konieczna et al. 2015), and we now show that changes in gene expression induced by Hcy-thiolactone, $\mathrm{N}$-Hcy-protein, and Hcy can also be involved.

The IPA analysis of pathways and networks based on gene expression results shows that Hcy-thiolactone strongly relates to 'CVD, cardiac infarction, skeletal, and muscular systems' among others (Table 6; Fig. 4). This is in line with the commonly reported manifestations of the HHcy and suggests the involvement of Hcy-thiolactone in those pathologies. This network (Table 6; Fig. 4) contains genes involved in chromatin organization/modification, which suggests important role of those genes in heart pathology induced by HHcy. Top network identified for $\mathrm{N}$-Hcy-protein involves genes affecting 'cardiovascular system development and function, dermatological disease, and conditions' (Table 6; Fig. 5), which suggests that $\mathrm{N}$-Hcy-protein contributes to heart and skin pathologies observed in HHcy. Top network identified for Hcy involves genes affecting 'amino acid and lipid metabolism' (Table 6; Fig. 6). This is consistent with previous studies that demonstrated the deregulation of amino acid (Akahoshi et al. 2014) and lipid (Maclean et al. 2012; Werstuck et al. 2001) metabolism in HHcy in mouse models.

HHcy is known to cause pathology in essentially all organs, including the cardiovascular and central nervous systems (Mudd et al. 2001). These pathologies are initiated by endothelial cell dysfunction and damage (Dayal and Lentz 2008). Thus, it may not be unexpected that exposure of HUVECs to individual metabolites such as $\mathrm{N}$-Hcyprotein, Hcy-thiolactone, and Hcy affects the expression of genes involved in neurological disease, ischemic disease, congenital heart disease, Alzheimer's disease, and neural tube defects.

In conclusion, our findings represent the first direct evidence that each Hcy metabolite uniquely modulates gene expression in molecular pathways and biological networks important for vascular homeostasis and identifies new genes and metabolic pathways linked to CVD. We also demonstrate that Hcy-thiolactone and $\mathrm{N}$-Hcy-protein, rather than Hcy, affect the majority of genes. One of the pathways containing genes affected only by Hcy-thiolactone is involved in chromatin/histone modifications. Thus, our findings suggest that alterations in chromatin modification gene expression might contribute to endothelial dysfunction and provide a possible mechanistic link between each metabolite and atherothrombosis. In addition, our findings underscore the importance of examining histone modifications in the context of HHcy-induced pathologies in humans.

Acknowledgments This work was supported in part by grants from the National Science Center, Poland, (2011/01/B/ NZ1/03417, 2011/02/A/NZ1/00010, 2012/07/B/NZ7/01178, 2013/09/B/NZ5/02794, 2013/11/B/NZ1/00091), MNiSW, Poland (N401065321504, NN302434439), and the American Heart Association (0855919D, 12GRNT9420014).

Conflict of interest The authors declare that they have no conflict of interest.

Open Access This article is distributed under the terms of the Creative Commons Attribution License which permits any use, distribution, and reproduction in any medium, provided the original author(s) and the source are credited.

\section{References}

Akahoshi $\mathrm{N}$ et al (2014) Neutral aminoaciduria in cystathionine betasynthase-deficient mice; an animal model of homocystinuria. Am J Physiol Renal Physiol 306:F1462-F1476. doi:10.1152/ ajprenal.00623.2013 
Albertini E, Koziel R, Durr A, Neuhaus M, Jansen-Durr P (2012) Cystathionine beta synthase modulates senescence of human endothelial cells. Aging 4:664-673

Benjamini Y, Hochberg Y (1995) Controlling the false discovery rate - a practical and powerful approach to multiple testing. J R Stat Soc B Met 57:289-300

Castro $\mathrm{R}$ et al (2005) Intracellular $S$-adenosylhomocysteine increased levels are associated with DNA hypomethylation in HUVEC. J Mol Med 83:831-836. doi:10.1007/s00109-005-0679-8

Chaturvedi P, Kalani A, Givvimani S, Kamat PK, Familtseva A, Tyagi SC (2014) Differential regulation of DNA methylation versus histone acetylation in cardiomyocytes during HHcy in vitro and in vivo: an epigenetic mechanism. Physiol Genomics 46:245255. doi:10.1152/physiolgenomics.00168.2013

Chwatko G, Boers GH, Strauss KA, Shih DM, Jakubowski H (2007) Mutations in methylenetetrahydrofolate reductase or cystathionine beta-synthase gene, or a high-methionine diet, increase homocysteine thiolactone levels in humans and mice. Faseb J 21:1707-1713

Corradetti MN, Inoki K, Guan KL (2005) The stress-inducted proteins RTP801 and RTP801L are negative regulators of the mammalian target of rapamycin pathway. J Biol Chem 280:97699772. doi: 10.1074/jbc.C400557200

Cuaz-Perolin C et al (2004) REDD2 gene is upregulated by modified LDL or hypoxia and mediates human macrophage cell death. Arterioscler Thromb Vasc Biol 24:1830-1835. doi:10.1161/01. ATV.0000142366.69080.c3

Dayal S, Lentz SR (2008) Murine models of hyperhomocysteinemia and their vascular phenotypes. Arterioscler Thromb Vasc Biol 28:1596-1605. doi:10.1161/ATVBAHA.108.166421

Den Heijer M, Lewington S, Clarke R (2005) Homocysteine, MTHFR and risk of venous thrombosis: a meta-analysis of published epidemiological studies. J Thromb Haemost 3:292-299. doi:10.1111/j.1538-7836.2005.01141.x

Devlin AM, Bottiglieri T, Domann FE, Lentz SR (2005) Tissue-specific changes in $\mathrm{H} 19$ methylation and expression in mice with hyperhomocysteinemia. J Biol Chem 280:25506-25511

DiBello PM, Dayal S, Kaveti S, Zhang D, Kinter M, Lentz SR, Jacobsen DW (2010) The nutrigenetics of hyperhomocysteinemia: quantitative proteomics reveals differences in the methionine cycle enzymes of gene-induced versus diet-induced hyperhomocysteinemia. Mol Cell Proteomics MCP 9:471-485. doi:10.1074/ mcp.M900406-MCP200

Dong E, Guidotti A, Grayson DR, Costa E (2007) Histone hyperacetylation induces demethylation of reelin and $67-\mathrm{kDa}$ glutamic acid decarboxylase promoters. Proc Natl Acad Sci USA 104:4676-4681. doi:10.1073/pnas.0700529104

Ellman GL (1959) Tissue sulfhydryl groups. Arch Biochem Biophys 82:70-77

Fiskerstrand T, Christensen B, Tysnes OB, Ueland PM, Refsum H (1994) Development and reversion of methionine dependence in a human glioma cell line: relation to homocysteine remethylation and cobalamin status. Cancer Res 54:4899-4906

Glowacki R, Jakubowski H (2004) Cross-talk between Cys34 and lysine residues in human serum albumin revealed by $\mathrm{N}$-homocysteinylation. J Biol Chem 279:10864-10871

Gough PJ, Greaves DR, Gordon S (1998) A naturally occurring isoform of the human macrophage scavenger receptor (SR-A) gene generated by alternative splicing blocks modified LDL uptake. J Lipid Res 39:531-543

Han JM et al (2012) Leucyl-tRNA synthetase is an intracellular leucine sensor for the mTORC1-signaling pathway. Cell 149:410 424. doi:10.1016/j.cell.2012.02.044

Handy DE, Castro R, Loscalzo J (2011) Epigenetic modifications: basic mechanisms and role in cardiovascular disease. Circulation 123:2145-2156. doi:10.1161/CIRCULATIONAHA.110.956839
Hortin GL, Seam N, Hoehn GT (2006) Bound homocysteine, cysteine, and cysteinylglycine distribution between albumin and globulins. Clin Chem 52:2258-2264. doi:10.1373/ clinchem.2006.074302

Ingrosso D et al (2003) Folate treatment and unbalanced methylation and changes of allelic expression induced by hyperhomocysteinaemia in patients with uraemia. Lancet 361:1693-1699

Jacovina AT et al (2009) Homocysteine inhibits neoangiogenesis in mice through blockade of annexin A2-dependent fibrinolysis. J Clin Investig 119:3384-3394. doi:10.1172/JCI39591

Jakubowski H (1995) Proofreading in vivo. Editing of homocysteine by aminoacyl-tRNA synthetases in Escherichia coli. J Biol Chem 270:17672-17673

Jakubowski H (1997a) Metabolism of homocysteine thiolactone in human cell cultures. Possible mechanism for pathological consequences of elevated homocysteine levels. J Biol Chem 272:1935-1942

Jakubowski H (1997b) Synthesis of homocysteine thiolactone in normal and malignant cells. In: Rosenberg IH, Graham I, Ueland PM, Refsum H (eds) Homocysteine metabolism: from basic science to clinical medicine. Kluwer Academic Publishers, Norwell, pp 157-165

Jakubowski H (1999) Protein homocysteinylation: possible mechanism underlying pathological consequences of elevated homocysteine levels. Faseb J 13:2277-2283

Jakubowski H (2000) Calcium-dependent human serum homocysteine thiolactone hydrolase. A protective mechanism against protein $N$-homocysteinylation. J Biol Chem 275:3957-3962

Jakubowski H (2002a) The determination of homocysteine-thiolactone in biological samples. Anal Biochem 308:112-119

Jakubowski H (2002b) Homocysteine is a protein amino acid in humans. Implications for homocysteine-linked disease. J Biol Chem 277:30425-30428

Jakubowski H (2007) The molecular basis of homocysteine thiolactone-mediated vascular disease. Clin Chem Lab Med 45:1704-1716

Jakubowski H (2008) The pathophysiological hypothesis of homocysteine thiolactone-mediated vascular disease. J Physiol Pharmacol 59(Suppl 9):155-167

Jakubowski H (2011) Quality control in tRNA charging—editing of homocysteine. Acta Biochim Pol 58:149-163

Jakubowski H (2012) Quality control in tRNA charging. Wiley Interdiscip Rev RNA 3:295-310. doi:10.1002/wrna.122

Jakubowski H (2013) Homocysteine in protein structure/function and human disease-chemical biology of homocysteine-containing proteins. Springer, Wien

Jakubowski H, Goldman E (1993) Synthesis of homocysteine thiolactone by methionyl-tRNA synthetase in cultured mammalian cells. FEBS Lett 317:237-240

Jakubowski H, Boers GH, Strauss KA (2008) Mutations in cystathionine beta-synthase or methylenetetrahydrofolate reductase gene increase $\mathrm{N}$-homocysteinylated protein levels in humans. Faseb J 22:4071-4076. doi:10.1096/fj.08-112086

Jakubowski $\mathrm{H}$ et al (2009) Genetic or nutritional disorders in homocysteine or folate metabolism increase protein $\mathrm{N}$-homocysteinylation in mice. Faseb J 23:1721-1727. doi:10.1096/ fj.08-127548

Jakubowski H, Zhang L, Bardeguez A, Aviv A (2000) Homocysteine thiolactone and protein homocysteinylation in human endothelial cells: implications for atherosclerosis. Circ Res 87:45-51

Jamaluddin MD et al (2007) Homocysteine inhibits endothelial cell growth via DNA hypomethylation of the cyclin A gene. Blood 110:3648-3655. doi:10.1182/blood-2007-06-096701

Joseph J, Loscalzo J (2013) Methoxistasis: integrating the roles of homocysteine and folic acid in cardiovascular pathobiology. Nutrients 5:3235-3256. doi:10.3390/nu5083235 
Kim CS et al (2011) Homocysteine promotes human endothelial cell dysfunction via site-specific epigenetic regulation of p66shc. Cardiovasc Res 92:466-475. doi:10.1093/cvr/cvr250

Kokame K, Kato H, Miyata T (1996) Homocysteine-respondent genes in vascular endothelial cells identified by differential display analysis. GRP78/BiP and novel genes. J Biol Chem 271:29659-29665

Konieczna A, Szczepanska A, Sawiuk K, Lyzen R, Wegrzyn G (2015) Enzymes of the central carbon metabolism: are they linkers between transcription, DNA replication, and carcinogenesis? Med Hypotheses 84:58-67. doi:10.1016/j.mehy.2014.11.016

Lentz SR (2005) Mechanisms of homocysteine-induced atherothrombosis. J Thromb Haemost 3:1646-1654

Maclean KN et al (2012) Cystathionine protects against endoplasmic reticulum stress-induced lipid accumulation, tissue injury, and apoptotic cell death. J Biol Chem 287:31994-32005. doi:10.1074/jbc.M112.355172

Mudd SH et al (1985) The natural history of homocystinuria due to cystathionine beta-synthase deficiency. Am J Hum Genet 37:1-31

Mudd SH, Levy HL, Kraus JP (2001) Disorders of transsulfuration. In: Scriver CR, Beaudet AL, Sly WS, Valle D, Childs B, Kinzler KW, Vogelstein B (eds) The metabolic and molecular bases of inherited disease, vol 2, 8th edn. Mc Graw-Hill, New York, pp 2007-2056

Nada S, Hondo A, Kasai A, Koike M, Saito K, Uchiyama Y, Okada M (2009) The novel lipid raft adaptor p 18 controls endosome dynamics by anchoring the MEK-ERK pathway to late endosomes. EMBO J 28:477-489. doi:10.1038/emboj.2008.308

Perla-Kajan $\mathbf{J}$ et al (2008) Immunohistochemical detection of $\mathrm{N}$-homocysteinylated proteins in humans and mice. Biomed Pharmacother 62:473-479. doi:10.1016/j.biopha.2008.04.001

Perla-Kajan J, Twardowski T, Jakubowski H (2007) Mechanisms of homocysteine toxicity in humans. Amino Acids 32:561-572

Plazar N, Jurdana M (2010) Hyperhomocysteinemia and the role of B vitamins in cancer. Radiol Oncol 44:79-85. doi:10.2478/ v10019-010-0022-Z

Pogribny IP, Karpf AR, James SR, Melnyk S, Han T, Tryndyak VP (2008) Epigenetic alterations in the brains of Fisher 344 rats induced by long-term administration of folate/methyl-deficient diet. Brain Res 1237:25-34. doi:10.1016/j.brainres.2008.07.077

Refsum H et al (2006) The Hordaland Homocysteine Study: a community-based study of homocysteine, its determinants, and associations with disease. J Nutr 136:1731S-1740S

Sauls DL, Lockhart E, Warren ME, Lenkowski A, Wilhelm SE, Hoffman M (2006) Modification of fibrinogen by homocysteine thiolactone increases resistance to fibrinolysis: a potential mechanism of the thrombotic tendency in hyperhomocysteinemia. Biochemistry 45:2480-2487

Sauls DL, Warren M, Hoffman M (2011) Homocysteinylated fibrinogen forms disulfide-linked complexes with albumin. Thromb Res. doi:10.1016/j.thromres.2011.01.009

Schmidt MT, Handschuh L, Zyprych J, Szabelska A, Olejnik-Schmidt AK, Siatkowski I, Figlerowicz M (2011) Impact of DNA microarray data transformation on gene expression analysiscomparison of two normalization methods. Acta Biochim Pol 58:573-580

Schnyder G et al (2001) Decreased rate of coronary restenosis after lowering of plasma homocysteine levels. N Engl J Med 345:1593-1600. doi:10.1056/NEJMoa011364

Sengupta $S$ et al (2001) Albumin thiolate anion is an intermediate in the formation of albumin-S-S-homocysteine. J Biol Chem 276:30111-30117
Sharma P, Senthilkumar RD, Brahmachari V, Sundaramoorthy E, Mahajan A, Sharma A, Sengupta S (2006) Mining literature for a comprehensive pathway analysis: a case study for retrieval of homocysteine related genes for genetic and epigenetic studies. Lipids Health Dis 5:1. doi:10.1186/1476-511X-5-1

Sikora M, Jakubowski H (2009) Homocysteine editing and growth inhibition in Escherichia coli. Microbiology 155:1858-1865. doi:10.1099/mic.0.026609-0

Sikora M, Marczak L, Kubalska J, Graban A, Jakubowski H (2014) Identification of $\mathrm{N}$-homocysteinylation sites in plasma proteins. Amino Acids 46:235-244. doi:10.1007/s00726-013-1617-7

Smyth GK (2004) Linear models and empirical bayes methods for assessing differential expression in microarray experiments. Stat Appl Genet Mol Biol 3: Article 3. doi: $10.102202 / 1544-61151027$

Smyth GK, Speed T (2003) Normalization of cDNA microarray data. Methods 31:265-273

Strauss KA et al (2007) Prevention of brain disease from severe 5, 10-methylenetetrahydrofolate reductase deficiency. Mol Genet Metab 91:165-175

Suszynska-Zajczyk J, Luczak M, Marczak L, Jakubowski H (2014a) Hyperhomocysteinemia and bleomycin hydrolase modulate the expression of mouse brain proteins involved in neurodegeneration. J Alzheimers Dis 40:713-726. doi:10.3233/JAD-132033

Suszynska-Zajczyk J, Sikora M, Jakubowski H (2014b) Paraoxonase 1 deficiency and hyperhomocysteinemia alter the expression of mouse kidney proteins involved in renal disease. Mol Genet Metab 113:200-206. doi:10.1016/j.ymgme.2014.07.011

Suszynska-Zajczyk J, Utyro O, Jakubowski H (2014c) Methionineinduced hyperhomocysteinemia and bleomycin hydrolase deficiency alter the expression of mouse kidney proteins involved in renal disease. Mol Genet Metab 112:339-346. doi:10.1016/j. ymgme.2014.05.010

Suszynska-Zajczyk J, Wroblewski J, Utyro O, Luczak M, Marczak L, Jakubowski H (2014d) Bleomycin hydrolase and hyperhomocysteinemia modulate the expression of mouse proteins involved in liver homeostasis. Amino Acids 46:1471-1480. doi:10.1007/ s00726-014-1712-4

Teslovich TM et al (2010) Biological, clinical and population relevance of 95 loci for blood lipids. Nature 466:707-713. doi:10.1038/nature09270

Tessarz P, Kouzarides T (2014) Histone core modifications regulating nucleosome structure and dynamics. Nat Rev Mol Cell Biol 15:703-708. doi:10.1038/nrm3890

Werstuck GH et al (2001) Homocysteine-induced endoplasmic reticulum stress causes dysregulation of the cholesterol and triglyceride biosynthetic pathways. J Clin Invest 107:1263-1273

Williams SR et al (2014) Genome-wide meta-analysis of homocysteine and methionine metabolism identifies five one carbon metabolism loci and a novel association of ALDH1L1 with ischemic stroke. PLoS Genet 10:e1004214. doi:10.1371/journal. pgen. 1004214

Yi-Deng J, Tao S, Hui-Ping Z, Jian-Tuan X, Jun C, Gui-Zhong L, Shu-Ren W (2007) Folate and ApoE DNA methylation induced by homocysteine in human monocytes. DNA Cell Biol 26:737744. doi:10.1089/dna.2007.0619

Zhao JY et al (2012) A functional variant in the cystathionine betasynthase gene promoter significantly reduces congenital heart disease susceptibility in a Han Chinese population. Cell Res. doi:10.1038/cr.2012.135 